\title{
A PROJECTED FINITE ELEMENT UPDATE METHOD FOR INVERSE IDENTIFICATION OF MATERIAL CONSTITUTIVE PARAMETERS IN TRANSVERSELY ISOTROPIC LAMINATES
}

Muhammad Zeeshan Siddiqui ${ }^{a}$, Sohaib Zia Khan ${ }^{a, b}$, Muhammad Ali Khan ${ }^{c}$, Kamran Ahmed Khan $^{\text {d, Majid Shahzad }}{ }^{\mathrm{e}}$, Salman Nisar ${ }^{\mathrm{a}}$, Danish Noman ${ }^{\mathrm{e}}$

${ }^{a}$ Department of Engineering Sciences, PN Engineering College, National University of Sciences and Technology (NUST), Karachi, Pakistan

${ }^{b}$ Department of Mechanical Engineering, Faculty of Engineering, Islamic University of Madinah, Madinah, Kingdom of Saudi Arabia

'School of Aerospace, Transport and Manufacturing, Cranfield University, Cranfield, UK

${ }^{d}$ Department of Aerospace Engineering, Khalifa University of Science, Technology and Research (KUSTAR), Abu Dhabi, UAE

'Space and Upper Atmosphere Research Commission (SUPARCO), Karachi, Pakistan

Abstract: In this paper, a novel application of Finite Element Update Method (FEUM) is proposed for the inverse identification of material constitutive parameters in transversely isotropic laminates. Two-dimensional Digital Image Correlation (2D-DIC) is used for full-field measurements which is required for the identification process. Instead of measuring the inplane displacements, which is a well-known application of 2D-DIC, we seek to measure the pseudo-displacements resulting from out-of-plane (towards camera) deflection of plate under a point load. These pseudo-displacements are basically the perspective projection of the three dimensional displacement fields on the image-plane of the image acquisition system. The cost function in this method is defined in terms of these projections instead of the true displacements - and hence the name Projected Finite Element Update Method (PFEUM). In this article, identification of in-plane elastic moduli of Carbon Fiber Reinforced Plastic (CFRP) plate has been performed using plate bending experiments which show predominantly out-of-plane deflection with little contribution from the in-plane displacements. Identification results are validated by direct experimental measurements of the unknown 
elastic constants as well as theoretical estimates based on volume ratio of constituents. The results show good conformance between estimated and target values for at least three material parameters namely $E_{1}, E_{2}$ and $G_{12}$. Effects of experimental noise on parameter estimates has also been evaluated to explain the observed deviation in estimated parameters with current test configuration.

Keywords: 2D field measurements; Inverse identification; Finite Element Model Update method; Out-of-plane motion; Perspective projection; Cost function minimization

\section{Corresponding author contact:}

Muhammad Zeeshan Siddiqui

Email: zeeshan@pnec.nust.edu.pk

Ph.: 0092-313-4313300 Fax: N/A

Address: PG Block, PNEC Campus, National University of Sciences and Technology, Karachi, 75350 


\section{Introduction}

In a typical problem in Solid Mechanics, often referred to as a direct or forward problem, the goal is generally to find the system's response (usually displacements) to known excitations (loads) assuming the geometry, constitutive parameters and displacement boundary conditions are known. In case of identification problems in solid mechanics, the available information generally pertains to the kinematic (displacement) field measurement made over part of the system boundary. The objective then is to find some of the characteristic parameters of the system by solving the physical system in an inverse manner - the inverse problem [1-3].

Due to the ever increasing computation power of modern computers as well as the developments in digital imaging devices, experiments rich in surface displacement information can easily be conducted. Furthermore, developments in full-field measurement techniques have made available a wealth of information about the deformation behavior of test specimens which are often heterogeneous in nature. Availability of heterogeneous displacement fields has motivated the researchers to relax the homogeneity requirement posed by the classical identification methods and develop, instead, novel inverse methods which make use of displacement field heterogeneity. Due to their diversity and robustness, the inverse identification methods have gained significant attention in last few decades [4-5]. The inverse methods may be classified according to their identification approaches; these include the model update methods like Finite Element Update Method (FEUM) $[2,6,7-8]$ and the Constitutive Relation Error method [8]. Another class of inverse methods is specifically based on satisfaction of equilibrium equations. The Constitutive Equilibrium Gap Method (CEGM) $[2,9]$ enforces equilibrium in a local form while the Virtual Fields Method (VFM) $[2-4,10]$ is derived from weak form of equilibrium equations. The Reciprocity Gap Method on the other hand mainly concerns situations where the field measurements are available at the boundary [8]. For a more detailed review of the inverse methods refer to Avril et al [11]. 
Most of the inverse identification techniques in solid mechanics rely on some form of image matching is one such tool which has been around for last few decades and has become one of the most popular displacement measurement techniques [12-14]. The image acquisition, required for extracting surface deformation information, is contact-less and tolerant to harsh environments. This makes the technique suitable for indoor as well as outdoor experimentation without interfering with the kinematic response of the object under observation. Potential drawbacks of this technique are due to the inherent noise and error sources present within the system. These include image sensor noise which may be dependent on temperature, the imperfections of optical lenses that are used for image capturing and local changes in contrast and brightness within an image [2]. The basic mechanism of image matching in DIC is the comparison of selected image subsets around a predefined set of points across two images taken before and after deformation. Modern DIC algorithms make use of the underlying deformation behavior of the deforming object to accelerate the matching process and improve measurement accuracy [7].

In Two-Dimensional Digital Image Correlation (2D-DIC), a single camera is used for taking images and is placed such that the optical axis of the camera is parallel to the outward normal of the surface under consideration. Compared to Three-Dimensional Digital Image Correlation (3D-DIC), where two cameras are used in a stereo arrangement, this method has benefits like ease of setup, less requirement of computation power as well as ease of calibration. Because of the simplicity and cost benefit that it offers, 2D-DIC is employed in a large number of applications in solid mechanics. The 2DDIC specimens are generally flat and subjected to in-plane loading (tension, compression, shear, biaxial or a combination of these loading conditions).

Traditionally, a limiting condition for the use of 2D-DIC is that the specimen surface and its subsequent deformation must lie within a plane $[12,15-16]$. The out-of-plane motion of the specimen, if any, is 
assumed to be negligible. In case the out-of-plane deformations are significant, the computed perspective nature of the conventional imaging systems. Use of telecentric lens has been shown to minimize these artificial displacements but is limited due to high cost of equipment, magnification and depth of field limitations $[6,15,17]$.

Detailed analytical study of the effect of general out-of-plane motion of the specimen - including rigid body motion - on the computed displacement field by 2D-DIC is given in $[6,17]$. By the new description proposed by the authors, 2D-DIC computes the difference between perspective projection of the specimen surface before and after deformation hence giving a two dimensional projection of the actual three dimensional displacement field. This means that even in the presence of large out-ofplane motion, the computed projection may still be useful and need not necessarily be termed as an undesirable effect. Although it is not possible, in general, to recover the true displacements, this projected displacement field can be used in some novel applications if (1) the projection of specimen's deformation state is unique and (2) the projection of its rigid-body motion, if present, is distinguishable from the projection of the underlying deformation. This research shows that uniqueness of the projected displacement field, which lies on the object plane of the imaging system, can be ensured provided the specimen geometry and loading conditions meet certain requirements like higher order continuity and higher degree of heterogeneity of the displacement field [17].

In this article, we use the Projected Finite Element Update Method (PFEUM) for inverse identification of material constitutive parameters for a Carbon Fiber Reinforced Plastic (CFRP) plate. The approach was first proposed by us in [17] where the method was used for a plate bending experiment with thin Aluminum plate made from AA5251 alloy with arbitrary rigid body motion. The technique involves comparison of perspective projection of out-of-plane deflection in a plate bending experiment with a corresponding numerical displacement field for defining a cost function. The material parameter 
estimation is then achieved by minimizing this cost function within elasticity constraints.

The minimization problem as well as composite modeling has been paid especial attention. Detailed experimental validation of the estimated parameters is done using direct measurement of material parameters by using conventional tensile tests. The estimated parameters with PFEUM show encouraging results as three of the four in-plane elastic parameters $\left(E_{1}, E_{2}\right.$ and $\left.G_{12}\right)$ show good conformance with the target values.

\section{Theory}

\subsection{Finite Element Update Method}

Among the commonly used inverse methods the most intuitive and popular method is the Finite Element Update Method (FEUM) [1-2]. Generally, a cost function defined in terms of numerical and experimental displacement fields is minimized on part of the system boundary in an iterative manner by changing the material constitutive parameters. Given a unique set of system geometry, material constitutive parameters, traction and displacement boundary conditions, the displacement response of a system is also unique [17]. Hence, assuming the system geometry and boundary conditions are correctly replicated in the Finite Element (FE) model, convergence between numerical and experimental displacement fields is achieved only when the constitutive parameters approach their true values.

In practice, however, experimental noise in the measured displacement field coupled with large number of material parameters (fully anisotropic material) may cause convergence difficulties and result in local minima during the minimization process. Even in the most favorable scenarios, a finite band around the true values will always be found in which the material parameters will vary. In either case, the test configuration may be carefully tailored to improve convergence $[6,17]$.

A brief theoretical development of the generic inverse approach is reproduced here from $[2,4,6-7]$. We assume an arbitrarily shaped linear elastic body $\Omega$ bounded by an external surface $S$ and having a 
volume $\mathrm{V}$ as depicted in Figure 1 . The boundary conditions are specified in terms of prescribed tractions on $\mathrm{S}_{\mathrm{T}}$ and prescribed displacements on $\mathrm{S}_{\mathrm{u}}$. Equations (1-3) are the governing equations for the system [4]

\section{Equilibrium equations}

$$
\left\{\begin{array}{l}
\operatorname{div}(\sigma)=0 \quad \text { in } \Omega \\
\sigma \cdot \mathbf{n}=\overline{\mathbf{T}} \quad \text { on } \mathrm{S}_{\mathrm{T}}
\end{array}\right.
$$

\section{Kinematic compatibility equations}

$$
\left\{\begin{array}{l}
\varepsilon=\frac{1}{2}\left[\operatorname{grad}(\mathbf{u})+\operatorname{grad}^{\mathrm{T}}(\mathbf{u})\right] \quad \text { in } \Omega \\
\mathbf{u}=\overline{\mathbf{u}} \quad \text { on } \mathrm{S}_{\mathbf{u}}
\end{array}\right.
$$

\section{Constitutive equations}

$$
\sigma=C: \varepsilon \quad \text { in } \Omega
$$

Where $C$ is the Hooke or elasticity tensor, $\sigma$ and $\epsilon$ represent the stress and strain tensors, $\mathbf{u}$ is the (unknown) displacement field in $\Omega, \mathbf{T}$ is the traction vector while $\mathbf{n}$ is the unit normal at any point on the surface with prescribed traction. The bar on $\mathbf{u}$ and $\mathbf{T}$ indicate prescribed quantities. We let $\mathrm{p}$ be $\mathrm{a}$ vector containing all the material parameters defining the constitutive behavior as

$$
\mathrm{p}=\left\{\mathrm{p}_{1}, \mathrm{p}_{2}, \mathrm{p}_{3}, \ldots, \mathrm{p}_{\mathrm{N}}\right\}
$$

Where $p_{1}$ to $p_{N}$ are constitutive parameters and $N$ is the number of unknowns in $p$ such that

$$
C=C(\mathrm{p})
$$

In inverse problems, a displacement field $\tilde{u}$ is experimentally measured on $\Gamma$ which is a subset of the system boundary $S$ as shown in Figure 1, while the elasticity tensor $C$ is unknown. Table 1 shows the difference between direct and inverse problems in terms of the known and unknown quantities. For 
material identification with FEUM, a solution for the system displacement response is available from function $S(p)$ can be defined over $\Gamma$, relating the numerical displacement field $\mathbf{u}$ with the experimentally measured displacement field $\widetilde{\mathbf{u}}$. The form of error function used in an inverse problem is not limited. A commonly used error function is the absolute integral error which may be written as $\mathrm{S}(\mathrm{p})=[\mathbf{u}-\tilde{\mathbf{u}}]^{\mathrm{T}}[\mathbf{u}-\tilde{\mathbf{u}}] \quad$ on $\Gamma$

$S(p)$ is the summation of error (squared) between the two displacement fields defined over the surface under consideration. Having defined the cost function, the solution to the inverse problem is sought by minimizing $S(p)$ over $\Gamma$ to seek an optimal $p[7,10]$.

$$
\min _{p} S(p) \quad p \in P
$$

Where $\mathrm{P}$ is the set of all the permissible values for $\mathrm{p}$ that satisfy the governing equations.

\subsection{The Projected Finite Element Update Method (PFEUM)}

In inverse problems using displacement measurement from 2D-DIC, the removal of in-plane rigid body motion is a trivial problem as this mode is easily recovered within DIC algorithms $[6,12,15]$. The presence of out-of-plane motion of specimen surface whether due to out-of-plane rigid body motion or due to out-of-plane deflection of the specimen (as in case of plate bending) has generally been a great concern in these experiments as the computed displacement field is easily corrupted with artificial displacements. Although this effect is quantifiable it cannot be separated from the underlying response of the surface [17].

In PFEUM, we make use of the fact that these artificial displacements in 2D-DIC are only a perspective projection of the true displacement field formed on the object plane of the imaging system [6, 17]. The imaging system used in 2D-DIC can be idealized as a pinhole camera [12, 15]. When an object is placed in front of a thin lens, all light rays emanating from different points of an object pass through 
a single point called pinhole which is at the center of the lens. The object plane is placed at a fixed distance $Z$ from the lens with positive $Z$ direction defined to point away from the lens as shown in Figure 2. We define a Coordinate System (CS) at the pinhole center which serves as a global CS for the imaging system. Another CS is defined at image center which is the projection CS. Both CSs are positioned on the optical axis as shown in Figure 2. If a point initially on the object plane $M$ moves a distance $\Delta Z$ away from the lens to a point $P$, Figure 3 , its projection on the object plane $\mathrm{M}^{\prime \prime}$ can be determined by using equations (8) and (9) [17].

$\mathrm{X}^{\prime \prime}=\alpha \mathrm{X}$

$\mathrm{Y}^{\prime \prime}=\alpha \mathrm{Y}$

Where $\alpha$ is a scaling parameter and $X^{\prime \prime}, Y^{\prime \prime}$ are the coordinates of the point on image plane in the projection CS. The scaling parameter only depends on the initial object distance $Z$ and the out-of-plane displacement $\Delta Z$. Given a three dimensional numerical displacement field $\mathbf{u}$, from FEA, the scaling parameter for each point in the displacement field can be found by equation (10)

$\alpha=\mathrm{Z} /(\mathrm{Z}+\Delta \mathrm{Z})$

Equations (8) through (10) can be used for obtaining a projected displacement field u" on the image plane. The out-of-plane rigid body modes - if present - are also compensated in this step by applying rigid-body transformations. For this reason, this step is called the out-of-plane motion (OPM) compensation. With the projected displacement field, we can redefine the cost function on the image plane by replacing the three dimensional numerical displacement field $u$ in equation (6) with $\mathbf{u}^{\prime \prime}$.

$S^{\prime \prime}(\mathrm{p})=\left[\mathbf{u}^{\prime \prime}-\widetilde{\mathbf{u}}\right]^{\mathrm{T}}\left[\mathbf{u}^{\prime \prime}-\widetilde{\mathbf{u}}\right] \quad$ on $\Gamma^{\prime \prime}$

Where $S^{\prime \prime}$ is the modified cost function, defined on $\Gamma^{\prime \prime}$ which is the projection of specimen surface on the object plane. It must be noted that $\widetilde{\mathbf{u}}$ is the projection of experimental displacement field and is 
already on $\Gamma^{\prime \prime}$. Hence, $\widetilde{\mathbf{u}}$ will be directly used in equation (11) to estimate the modified cost function while compensation for out-of-plane motion is only applied on $\mathbf{u}$ before taking projection. In the presence of rigid-body out-of-plane motion, the parameter vector $p$ in equation (11) will be embedded with three additional parameters defining the out-of-plane rigid body modes - an out-of-plane translation along $Z$ direction while two rotations about $X$ and $Y$ axes, respectively - which will be concurrently converged along with the material parameters. The schematic of the overall identification problem is depicted in Figure 4.

The difference between this approach and the classical FEUM approach is clearly noted. Instead of directly using the numerical displacement field from FEA, we take its projection on the image plane and use this projection for defining the cost function. Since both the numerical and experimental displacement fields are now only projections, we term this method as Projected Finite Element Update Method (PFEUM). Compared to the classical FEUM technique with 2D-DIC, the only additional step in PFEUM is the application of equations (8) and (9) to the numerical displacement field which is very straight forward. Not only does the requirement of suppressing or compensating the rigid-body motion apply equally to both methods, but the assumption of pin-hole camera model, for calculating projection, is also not new as the calibration required for 3D-DIC is totally based on this assumption [12]. With 3D- DIC, the noise level is generally higher due to added uncertainty arising from image matching across multiple cameras. Particularly for out-of-plane motion, the expected error is about 4 times higher than in-plane displacements (ref [12], pp 181). Furthermore, any camera movement or vibration post calibration will result in increasing uncertainty of the measured displacements; with PFEUM, however, it is possible to recover such errors as rigid body motion alongside the material parameters. The proposed approach hence offers a very attractive alternative to the 3D-DIC based FEUM which, apart from the additional cost of equipment and software, requires considerably more 
effort for setup and calibration, or in some cases may be inapplicable due to availability or accessibility constraints.

\section{Experiments}

In this research, we used this novel approach for inverse identification of material constitutive parameters of a CFRP plate using 2D-DIC. The composite plate was assumed to be transversely isotropic and hence the number of unknown material parameters was limited to four - two in-plane Young's moduli ( $E_{1}$ in fiber direction and $E_{2}$ in lateral direction), in-plane Poisson's ratio $\left(v_{12}\right)$ and inplane shear modulus $\left(\mathrm{G}_{12}\right)$. The plate was fixed at three or four points and would deflect out-of-plane under the application of a concentrated load. In general plate deflection for this case can be represented by equation (12) $[18,19]$.

$\mathrm{D}_{11} \frac{\partial^{4} \mathrm{w}}{\partial \mathrm{x}^{4}}+\left(\mathrm{D}_{12}+2 \mathrm{D}_{66}\right) \frac{\partial^{4} \mathrm{w}}{\partial \mathrm{x}^{2} \partial \mathrm{y}^{2}}+\mathrm{D}_{22} \frac{\partial^{4} \mathrm{w}}{\partial \mathrm{y}^{4}}=\mathrm{q}(\mathrm{x}, \mathrm{y})$

Where $w$ is the plate deflection, $q$ is the applied load and $x, y$ are the spatial coordinates of the plate. The orthotropic stiffness coefficients $D_{i j}$ may be defined by independent elastic constants and plate thickness $\mathrm{h}$ in the following manner,

$$
\mathrm{D}_{11}=\frac{\mathrm{E}_{1} \mathrm{~h}^{3}}{12\left(1-v_{12} v_{21}\right)}, \quad \mathrm{D}_{22}=\frac{\mathrm{E}_{2} \mathrm{~h}^{3}}{12\left(1-v_{12} v_{21}\right)}, \quad \mathrm{D}_{12}=v_{12} \mathrm{D}_{22}=v_{21} \mathrm{D}_{11}, \quad \mathrm{D}_{66}=\frac{\mathrm{G}_{12} \mathrm{~h}^{3}}{12}
$$

Where $v_{\mathrm{ij}}$ is the Poisson's ratio and $v 12 E 2=v 21$ for isotropic laminates.

\subsection{CFRP plates}

The CFRP plates for the experimentation were made using unidirectional tape of Carbon Fiber prepregs. The fiber yarn used for the prepreg manufacturing was TAIRYFIL TC-36S with $12 \mathrm{~K}$ filaments with a fiber diameter of $7 \mu \mathrm{m}$ [20]. Detailed manufacturer supplied properties of the carbon fibers are given in 
Table 2 2. The resin used in prepregs was SWANCOR 2552 epoxy with a density of $1.2 \mathrm{gm} / \mathrm{cm}^{3}$ in cured

form [21]. Table 3 shows the key characteristics of the resin as provided by the manufacturer.

The development of CFRP plate for our experiments was done by stacking 14 layers of unidirectional prepreg sheets in a $280 \mathrm{~mm} \times 270 \mathrm{~mm}$ mold. The mold was then placed in a hot press for 1.5 hours at $150{ }^{\circ} \mathrm{C}$ under 20 bar pressure resulting in a vertical load of $98 \mathrm{kN}$ on the composite surface. The resulting thickness of the composite plate was $1.95 \pm 0.05 \mathrm{~mm}$. Upon curing the 14 layers effectively fused together and were no more distinguishable from each other even on microscopic examination. After curing, a $200 \mathrm{~mm} \times 150 \mathrm{~mm}$ specimen was cut from the plate using HSS slitting cutter. No significant bending of the plate was observed during post cure cool-down or cutting operation, which was confirmed by a flatness of less than $0.5 \mathrm{~mm}$ measured by a Coordinate Measurement Machine (Premier, South Korea). This specimen was then painted white on both faces. Load application points were carefully marked on one side of the specimen (back face), while a fine speckle pattern of nonglossy black paint (matte finish, to minimize reflection during image acquisition) was applied on the front face for image correlation. Vertical and horizontal center lines were carefully marked on the speckle side to facilitate centering of the specimen and camera alignment as shown in Figure 5.

\subsection{Setup}

The experimental setup consisted of a test bench made from stainless steel. The brackets holding the specimen were $10 \mathrm{~mm}$ thick to ensure minimal support deformation. The specimen was held in position between pointed bolts at each support location as shown in Figure 6. The support points were at a distance of $5 \mathrm{~mm}$ from specimen edges as shown in the specimen schematic in Figure 7 . Since the supports had to be accurately reproduced in FE model for obtaining the numerical displacement field, pointed bolts were used to grip the specimen which can then be modeled by holding a single node at this location in FE model. Gripping force of less than $1 \mathrm{~N}$ between the two bolts was initially set. This small force was measured by placing a small $2 \mathrm{lb}$ load-cell (FUTEK, Germany) in place of one of the 
bolts and then calibrating the hand tightening method of the operator. The procedure was repeated each tightening. The purpose of using a controlled but small gripping force was to ensure contact of all supporting bolts on the front and back faces of the specimen while minimizing the chances of penetration of pointed bolts in the specimen surface. Upon application of initial load - which was applied on the back face, the bolts on the back face would become free and would not contribute any further reactions during experiment. The overall specimen arrangement is shown in Figure 8. The concentrated load at the plate center was applied by controlled rotation of a nut on $5 \mathrm{~mm}$ diameter threaded bolt. The arrangement was such that the rotation of nut caused the bolt to travel towards the plate. The magnitude of applied load was measured by an S-type FUTECK load cell with a maximum capacity of $45 \mathrm{~N}$ in compression.

Due to the inherent complexity in composite materials' modeling and experimentation, the test setup was made rigid enough to ensure negligible support deflections as well as rigid body motion. In our work, the supports were made with $10 \mathrm{~mm}$ thick stainless steel brackets rigidly mounted on a $13 \mathrm{~mm}$ thick base plate. A preliminary FE simulation of the support structure with maximum load encountered in this work showed negligible support deformations (about $1.2 \mu \mathrm{m}$ out-of-plane deflection at supports compared to about $1.5 \mathrm{~mm}$ plate deflection). The assumption of rigid supports then allowed skipping the rigid-body modes from the parameter vector $p$ in equation (11) and thus assisted in overcoming the convergence difficulties due to the additional material parameters as compared to the isotropic case. The simplification was motivated by $[4,10]$, where a similar test arrangement was used with the Virtual Fields Method while neglecting support deformations, to reduce the complexity and computation cost of each iteration.

For this arrangement the plate deflection, $w$ in equation (12), was along the camera's optical axis. Hence the observed displacement and its projection would both be fourth-order continuous giving 
enough heterogeneity of the displacement field to make it distinguishable from the projection of rigid body modes described in [17]. Heterogeneity of the displacement field may further be increased by disturbing the specimen symmetry as discussed later.

\subsection{0ptical system and Image correlation}

The camera used for image acquisition was a SONY XCG5005E monochrome camera with an image depth of 12 bits. The imaging sensor had an effective resolution of $2448 \times 2048$ pixels. The camera was fitted with a Schneider Optics high resolution lens having a variable focal length of $1.8 \mathrm{~mm}$ to 35 $\mathrm{mm}$. The front lens element to specimen front face distance was carefully measured to be $981 \mathrm{~mm}$. The perpendicularity of camera optical axis with specimen surface was ensured by aligning an image grid with the specimen center lines and a similar grid pattern drawn on the back wall facing the experimental setup. The images were calibrated by using specimen width along the centerline.

The experimental displacement field was measured by analyzing the captured images with 2D-DIC using an indigenously developed code (OSM) [22] in MATLAB ${ }^{\odot}$. The code uses a two-step search algorithm for displacement measurement with a coarse search using normalized cross-correlation followed by a non-linear search with the Levenberge-Marquardt algorithm. OSM uses bi-cubic interpolation for sub-pixel resolution and utilizes a normalized intensity matrix for correlation search to cater for the change in light intensity within and across images. Test results with this code using standard image sets provided by the Society of Experimental Mechanics [23] are given in appendix A. Due to very small expected magnitude of the measured displacements, five images were taken at each load step and then averaged to minimize the measurement noise due to various effects such as light intensity and main frequency variations. The images were further operated upon by a $15 \times 15$ pixel Gaussian filter before correlation. A relatively sparse correlation grid was selected with a subset spacing of 55 pixels to reduce computation time. Since high local strain gradients were not expected, a large subset size of 61 pixels was used to minimize correlation uncertainty. This combination resulted 
in a small overlap of 3 pixels. Under these conditions, the image correlation system gives a standard deviation of less than 0.01 pixels around the mean displacement value.

\subsection{Finite Element Model}

The finite element model of the bench was made with ANSYS APDL script using the 20-node SOLID186 brick elements. The choice between SOLID186 and SHELL281 was arbitrary as only $0.15 \%$ difference was observed between plate deflections for SHELL281 and SOLID186 elements under same element size, load and boundary conditions with one SOLID186 element across the thickness. A mesh convergence study was also carried out and it was found that below an element size of $10 \mathrm{~mm}$, there was no significant change in plate deflection. An element edge length of $10 \mathrm{~mm}$ was thereafter used for sizing of elements in FE mesh. The element coordinate systems were oriented such that the fibers were oriented length wise (parallel to the $200 \mathrm{~mm}$ edge).

\subsection{Case descriptions}

In this work, inverse identification study on the composite plate was done with two experimental cases having identical specimens but different force and displacement boundary conditions.

Case I: The plate was fixed at four corners as shown in Figure 6, while a concentrated force was applied at plate center.

Case II: The plate was fixed at three corners while the corner in $(X,-Y)$ quadrant was free. Point load was applied at $(-50,65) \mathrm{mm}$.

The test configuration in case II was inspired from [10], which showed the best parameter estimates with the VFM using grid method for curvature measurements.

\section{Validation Setup}

Before proceeding with the inverse characterization, the composite plate was characterized using classical test methods. This characterization was done to get reliable reference values for the inverse problem. 


\subsection{Rule of mixtures}

The elastic moduli were first calculated using the rule of mixtures. The percentages of resin and fiber in the composite were found by digesting the resin in 65\% nitric acid as per ASTM standard D3171 [24]. The weight percentages found from this method were then converted to volume percentages using reference densities of epoxy resin and carbon fiber using equation (14) and equation (15).

$\mathrm{V}_{\mathrm{f}}=\frac{\mathrm{d}_{\mathrm{c}}}{\mathrm{d}_{\mathrm{f}}} * \mathrm{~W}_{\mathrm{f}}$

$\mathrm{V}_{\mathrm{m}}=\frac{\mathrm{d}_{\mathrm{c}}}{\mathrm{d}_{\mathrm{m}}} * \mathrm{~W}_{\mathrm{m}}$

Where $V_{f}$ and $V_{m}$ are volume fractions of fiber and matrix, respectively, $W_{f}$ and $W_{m}$ are their weight fractions while $d_{c}, d_{f}$ and $d_{m}$ are the densities of composite, fiber and matrix, respectively. Density of the cured composite specimen was found to be $1.56 \mathrm{gm} / \mathrm{cm}^{3}$. Table 4 shows the estimated composition of the cured composite specimen. Internal structure of the composite was also investigated by using optical microscope. The cross-section of the composite specimen was prepared by fine grinding as shown in Figure 9. The image shows high compactness with small voids appearing as dark spots. The volume composition was also confirmed from the analysis of this image by our indigenous image analysis code OIT in Matlab ${ }^{\circledR}$. The code uses two sliders which allows the user to select a threshold band ( $h_{\min }$ to $h_{\max }$ ) encapsulating the grayscale values for the resin system. The pixels with grayscale values below $h_{\min }$ are assigned to the void content while those above $h_{\max }$ are assigned to the fibers. Once all pixels have been categorized, the code simply counts the total number of pixels in each category and calculates their percentages which are assumed to approximate the volume fractions. After analysis by this code, the fiber volume fraction and void content were found to be $60 \%$ and $1.5 \%$, respectively.

The properties of composite specimen were finally computed using rule of mixtures and the inverse rule of mixtures by equations (16) and (17), respectively. 
$E_{1}=E_{f} * V_{f}+E_{m} *\left(1-V_{f}\right)$

$E_{2}=\left(\frac{V_{f}}{E_{f}}+\frac{1-V_{f}}{E_{m}}\right)^{-1}$

Where $E_{1}$ is the fiber direction modulus, $E_{2}$ is the transverse modulus, $E_{f}$ and $E_{m}$ are corresponding elastic moduli for fiber and matrix and $V_{f}$ is the fiber volume fraction.

\subsection{Tensile tests}

Tensile tests of the composite specimens cut from similar composite panels were also conducted using ASTM standard D3039 test coupons for $0^{\circ}$ and $90^{\circ}$ unidirectional fiber orientations. Resistance type bonded strain gauges (Vishay, USA) with $6 \mathrm{~mm}$ gage length and $350 \Omega$ resistance were bonded on the specimen surface for measurement of axial and lateral strains. The specimens were tested on a 150 KN Tinius Olsen universal testing machine. The stress strain data was then used for computing three elastic moduli $\left(E_{1}, E_{2}\right.$ and $\left.v_{12}\right)$. Table 5 shows the measured values and compares the results obtained from rule of mixtures with tensile tests.

\section{Results and Discussion}

\subsection{Effect of statistical fiber misalignment}

During manufacturing of composite plates, the resin flow across the fiber direction as well as the inherent twist of fiber tows during manufacturing of prepregs cause local misalignment of the fiber orientations from the mean fiber direction. This phenomenon is present regardless of the number of physical prepreg layers present in the laminate. The resulting laminate has fibers forming a narrow distribution of angles around the mean fiber orientation which can affect the elastic moduli of the laminate [26]. This effect was studied by preparing a sample for microscopic examination with fine grinding. The surface to be examined was parallel to the plane of lamina. Hence, a clear picture of the orientation of individual fibers was revealed as shown in Figure 10. A total of 14 images were gathered at different locations. $A$ MATLAB ${ }^{\odot}$ code was used for detection of edges in the captured images using Sobel edge detection function with a threshold of 0.02 . The orientation of approximately 3800 fibers 
which were detected in these images is shown in Figure 11. After analysis of all images, the overall (horizontal) fiber direction. The probability distribution function for the total population of detected fibers is shown in Figure 12.

The distribution of the fiber orientation was also approximated in FEA for the comparison of maximum deflection in two different scenarios - both representing the plate described as case II in the previous section. The first scenario corresponds to a composite plate having alternate layers oriented at $\pm 2.3^{\circ}$ in the ply plane. In the second scenario, the composite plate has all fibers perfectly aligned with the $0^{\circ}$ mean orientation. The trend of difference between the two displacement fields, relative to the maximum deflection, with increasing number of layers is shown in Figure 13. It is seen from this result that if sufficiently large number of fibers (with each fiber modelled by a single layer) are present in the laminate across the thickness, the effect of local misalignment in fiber orientation will disperse as the maximum relative difference with 200 layers is only $2.5 \%$. Since the laminate is transversely isotropic, the orientation of fibers within a ply will follow the same pattern and with very high number of fibers along the length of plate, the effect will be even more dispersed. It is also noted here that for a filament diameter of $7 \mu \mathrm{m}$, as indicated in Table 2, about 278 fibers will be present across the thickness in a $1.95 \mathrm{~mm}$ thick plate. Modelling such a large number of fibers, with unique orientation, as individual layers is prohibitive due to computation cost occurred in each iteration of the inverse problem; particularly statistical modelling of the random local fiber orientations with in a layer is, to date, not possible in ANSYS APDL and hence an approximation has to be made. It is also noted that modelling randomly oriented layers at run time would likely throw the gradient-based optimization off-course if the fiber orientations change even slightly during the gradient calculations. Moreover, the difference in maximum plate deflection with 278 randomly oriented layers is only $2.28 \%$. The corresponding number for a plate under case I boundary conditions is only $0.46 \%$. This difference is 
much less than the expected magnitude of random noise present in the measured displacement field. For this reason, the fiber orientation was assumed to coincide with the mean layer orientation and the effect of local changes in fiber orientations was considered negligible. The effect of this fiber misalignment on mechanical properties of composites have been studied by Bednarcyk et el [26]. Their results indicate that the transverse elastic moduli $\left(E_{1}\right.$ and $\left.E_{2}\right)$ are not much affected by fiber misalignment. A misalignment of about $2.3^{\circ}$, as encountered in this study, reduced $E_{1}$ by less than $1 \%$ while an increase of same magnitude was observed in $E_{2}$. However, $G_{12}$ is more sensitive to these misalignments and an increase of about $8 \%$ was observed. No results for $v_{12}$ have been reported but it is expected that the change will be similar to $\mathrm{G}_{12}$. These results indicate that the expected target values for $\mathrm{G}_{12}$ will be a little higher than those reported here.

\subsection{Effect of noise}

Displacement measurements in 2D-DIC are prone to noise from a number of sources. These sources include light intensity fluctuation, noise from CCD sensor as well as the inherent uncertainty of the image correlation process $[2,12]$. Experiments were conducted to study the effect of noise in the measured displacement field on the accuracy of estimated parameters with the inverse problem. Numerical experiments were performed by using the FE model of the composite plate with known material parameters under a load of $15 \mathrm{~N}$. The numerical displacement field was then polluted by adding zero mean Gaussian noise with standard deviation ranging from $0 \%$ to $10 \%$ of the average inplane pseudo displacement field. This pseudo displacement field was obtained by projecting the numerical displacements on the image plane of a camera assumed to be placed at a distance of $1 \mathrm{~m}$ from the specimen surface. Figure 14 depicts the procedure adopted for noise addition to a sample displacement field with unit displacement at the center.

With the simulated experimental displacement field, $\tilde{u}$ computed as described above, the material parameters were identified for each noise level under case I and case II conditions using inverse 
procedure. The minimization problem was solved nine times for each noise level (with random noise Coefficient of Variation (CV) for each constitutive parameter is used to quantify successful convergence. A low $\mathrm{CV}$ value indicates smooth convergence to a unique parameter value while increasing CV will indicate convergence difficulties. The convergence pattern for case I with $3 \%$ noise is shown in Figure 15. It is clearly seen that convergence for the in-plane Poisson's Ratio $\left(v_{12}\right)$ is poor compared to other parameters. Figure 16 shows the CV computed for all material parameters as a function of increasing noise levels in case I, while Figure 17 plots the same for case II. The convergence of both cases without noise (the first point) is almost perfect and minimal variation from target values is observed. While it is evident that $\mathrm{v}_{12}$ is highly sensitive to noise for both case I and case II, the convergence of the other three parameters in case II is much more robust and stays below $5 \%$ even at a high noise level of $10 \%$.

It is also interesting to explore loading geometries which are sensitive to specific material parameters. For example, a beam in bending would show anticlastic deformation which is proportional to the inplane Poisson's ratio. Similarly, the in-plane shear modulus would dominate the displacement response of a beam under twisting load. The discussion of these geometries and results of simulated experiments exploring the test configurations are included in Appendix B.

\subsection{Inverse Identification}

The experimental identification for the two cases were carried out at 7 different loads ranging from 7 $\mathrm{N}$ to $22 \mathrm{~N}$ at $2 \mathrm{~N}$ intervals. Since the plate was not perfectly flat, an initial load of $5 \mathrm{~N}$ was applied to make sure the observed pseudo displacements were consistent with the load direction. Since the plate deflection was of the order of plate thickness, non-linear effects were also taken into account in the FE solutions. The FE solutions were performed in two steps; the first step pertains to the initial load while the second step corresponds to the final load. The displacements from the initial load were 
subtracted from the second load step before exporting the displacements from APDL. The computed noise levels. The difference between experimental displacements and the smoothing surface was considered to be noise. A noise level between $5 \%$ and $10 \%$ of the average displacement was found by this approach. For identification purpose, however, the raw displacement data was used to avoid uncertainties due to any lack of agreement between experimental displacements and smoothing surface. The initial guess for the optimization were selected randomly in $\pm 50 \%$ of the target range as shown in Table 7 and Table 8 for case I and case II, respectively. The bounds on elastic parameters during optimization (Table 6) were selected far away from the target location to check the possibility of local minima during convergence. This meant that if a parameter was identified very close to a bound, it was considered a convergence failure.

The convergence pattern at different loads for case I and II is shown in Figure 18 and Figure 19, respectively. The applied loads, initial guess and consolidated results for the two cases are shown in Table 7 and Table 8, respectively. The estimated parameters in case I show poor convergence for $\mathrm{v}_{12}$ and $\mathrm{G}_{12}$ as indicated by very high $C V$ values of $45.8 \%$ and $44.7 \%$, respectively. While $E_{1}$ and $E_{2}$ converge well for each load level, the relative error for $E_{2}(59.8 \%)$ is still very high compared to $E_{1}(4 \%)$. This result shows the test configuration is not well suited for the inverse method in its present form. The reason for this poor convergence is the symmetry of displacement field as a result of symmetric supports and load application at the center. A similar result was found in [4] for the VFM. When the displacement field is symmetric, Poisson's effect will cause coupling of $E_{1}$ with $E_{2}, v_{12}$ and $G_{12}$. For example, a deformation in $E_{1}$ direction will show up in $E_{2}$ due to Poisson's effect but in opposite direction. This will result in an increased apparent stiffness in $E_{2}$ direction which is also confirmed from the results for case $I$. This result is consistent with the noise study which shows $v_{12}$ and $G_{12}$ to be most sensitive to noise in case I. 
The parameter estimation for case II is in close agreement for $E_{1}, E_{2}$ and $G_{12}$ as depicted by low $C V$ target values found by direct measurement. However, $\mathrm{v}_{12}$ shows clear divergence for almost all load levels - this result again confirms with the findings of simulated noise study in previous section where it was found that $v_{12}$ is very sensitive to noise levels above $2 \%$.

Although an overall improvement in parameter estimation of case II over case I is clearly seen, the divergence of $v_{12}$ in case II means that even though the test configuration of case II was optimized for VFM [4], it is not an optimal configuration for PFEUM. The improvement in parameter identifiability in case II over case I is a result of lack of symmetry and hence increased heterogeneity of displacement field.

Reduced convergence with symmetric displacement field may also be attributed to the low sensitivity of displacement field for the individual parameters. Particularly, the sensitivity of the displacement field for $v_{12}$ seems to be low thus causing divergence at even very small noise levels.

In order to check the effect of initial guess on the convergence, the minimization problem was solved for case II at $18 \mathrm{~N}$ load ten time with different initial guesses. The initial guess for each run was again selected randomly as done earlier. The results showed a standard deviation of less than 0.006 for all parameters with the mean value exactly as shown in Table 8 . This shows that the convergence is largely independent of the initial guess and the target values could have been used equally well for initial guess without comprising the convergence. In this way the minimization problem could have been regularized and a faster convergence made possible.

\section{Conclusion}

Projected Finite Element Update Method (PFEUM) has been used for inverse identification of material constitutive parameters in a carbon fiber reinforced composite plate. Effect of experimental noise on material parameter identifiability has been studied by simulated experiments. The change of 
coefficient of variance in estimated parameters with respect to the amplitude of noise gives the expected range of accuracy for each parameter in current experimental setup.

PFEUM is then used for experimental estimation of constitutive parameters with inverse method. The approach is shown to produce encouraging results for at least three of the four identified parameters $\left(E_{1}, E_{2}\right.$ and $\left.G_{12}\right)$ for the case of CFRP plates. For the in-plane Poisson's ratio $\left(v_{12}\right)$, the convergence becomes increasingly difficult with increase in noise level. This is an expected result as the noise sensitivity analysis showed high variation for $v_{12}$ with noise levels above $2 \%$. The overall estimation of the other three parameters is well within acceptable limits and is further validated by direct experimental measurement of these parameters using classical test methods.

The performance of the 2D-DIC code has also been evaluated with test images provided by Society for Experimental Mechanics and results have been included. Similarly test configurations which are known to be sensitive to certain material parameters have been explored to study the identifiability of these parameters. The results indicate that a beam specimen with anticlastic curvature, as well as twist load can be used to accurately measure $\mathrm{G}_{12}$.

This study highlights the potential of PFEUM method of inverse characterization of composite materials as specimen preparation and testing procedure with this method is very simple and use of 2D-DIC for measurement of pseudo displacements can be easily accomplished. Future developments in this study will include a detailed optimization of test configuration with respect to different test parameters, geometry and fiber orientation. The authors will also attempt to quantify the sensitivity of the displacement field with respect to each parameter which will allow for an improved configuration design. 


\section{Acknowledgements}

The authors wish to thank Mr. Tasleem Baig and Dr. Ali Kamran for the permission to use experimental facilities. Additionally, the authors would like to acknowledge the support by Mr. Nizam Uddin Ahmed, Mr. Muhammad Tahir and the staff from manufacturing and material testing laboratories at SUPARCO.

\section{Appendix A}

The Society of Experimental Mechanics (SEM) has published standard image sets [23] for evaluation of both 2D/3D DIC codes and a number of researchers have already evaluated their DIC codes with these datasets. In this article, the first two image sets for 2D-DIC were analyzed using OSM and the results have been compared with corresponding results for Ncorr $[27,28]$ to give an idea about the accuracy and precision of the 2D-DIC results reported in this article. A subset size of 21 pixels was used for both datasets with a step size of 10 pixels. A $470 \times 470$ pixel area from the image center was selected for correlation resulting in 2304 points on which displacement was measured.

The comparison was based on two test criteria for evaluating the performance of the DIC algorithms with each dataset. First was the Average Displacement Error (ADE) which is defined as $\left.(<\mathbf{u}\rangle-\mathbf{u}_{\mathbf{0}}\right)$, where $\langle\mathbf{u}\rangle$ is the average measured displacement in the $\mathbf{X}$ direction and $\mathbf{u}_{\mathbf{0}}$ is the specified displacement. The ADE is a measure of global performance of the DIC code. A Local Displacement Error (LDE) was also specified as $\left[<\left(\mathbf{u}-\mathbf{u}_{\mathbf{0}}\right)^{2}>\right]^{1 / 2}$ which is the standard deviation of each measurement around the specified displacement value. The LDE provides estimate of the expected error on individual measurement points.

The first dataset (Sample 1) corresponds to varying contrast images with $1.5 \sigma$ grey level noise. The low noise results in good precision of measured displacements. Figure 20 shows the ADE and LDE for Sample 1 images processed with two different subset sizes of 21 and 31 pixels. For an ROI size of 21, the maximum $A D E$ in the measured displacements over the entire $48 \times 48$ grid points is $2.42 \mathrm{e}-4$ pixels, while the maximum LDE is $3.41 \mathrm{e}-3$ pixels. For the same subset size in Ncorr, the corresponding 
numbers for ADE and LDE are 3.2e-4 and 5e-3, respectively, which indicate that OSM results are slightly more accurate than Ncorr. Since the images have varying contrast, the LDE increases steadily as the image contrast is reduced but the ADE remains unchanged. As expected, both error norms reduce as the subset size is increased to 31 pixels.

The second dataset (Sample 2) has little contrast variation across images but has a very high image noise of $8.0 \sigma$ grey levels. The noise in input images results in high standard deviation of measured displacements. The ADE and LDE for Sample 2 images are shown in Figure 21. The maximum ADE and LDE values for this dataset are found to be 0.024 and 0.234 , respectively. The corresponding values for Ncorr are -0.036 and 0.54 . This comparison with Ncorr reveals that OSM results are again slightly more accurate. Since contrast variation across the images in Sample 2 is not significant the LDE remains rather constant. A significant improvement in LDE is observed as the subset size is increased to 31 .

Although comparison could not be made with VIC-2D, the commercial 2D-DIC software by Correlated Solutions ${ }^{\circledR}$ [29], comparison of Ncorr with VID-2D in [28] indicates that OSM also compares well in terms of accuracy with the commercial software. Detailed results for different samples in the dataset by OSM as well as tabulated data for comparison may be found in [30].

\section{Appendix B}

In view of the results of the noise study done in this article, it is clearly seen that a given set of specimen geometry and loading as well as boundary conditions may activate a certain material parameter which then dominates the deformation behavior under applied load. Hence it is insightful to explore geometries which are known to favor a certain material property. Here we report the results of the investigation that has been done to explore the merits of such geometries.

For example, when an elastic beam is subjected to a bending load, an anticlastic curvature is observed due to the Poisson's effect. This curvature is directly dependent on the value of in-plane Poisson's 
ratio. Simulated experiments similar to those for plate bending were designed to study this effect. Figure 22 shows a beam made of unidirectional CFRP material under bending load. The fibers were oriented along the axis of the beam which was parallel to the $X$ axis. Finite Element model of the plate was created in ANSYS APDL and a bending load was applied along the $Z$ direction and the resulting deformations of the beam face in the $Z$ direction were recorded. For such a loading condition, the beam would primarily develop a curvature about the $Y$ axis which in turn, due to the Poisson's effect, would create an anticlastic curvature about the $X$ axis - but in the opposite direction. Projection of the numerical displacement field found in this simulated experiment was taken on the image plane of the camera using equations (8) and (9). The sensitivity of material identification with this simulated field was then studied with different levels of added noise.

Figure 23(a) shows the estimated material parameter variation with increasing noise level. Although it was expected that the anticlastic curvature would strongly favor identification of the in-plane Poisson's ratio $v_{12}$, the results indicate that only the fiber direction modulus $E_{1}$ may be identified with any reasonable accuracy once the noise level is greater than $5 \%$. However, an interesting effect is seen when the fibers are oriented at $5^{\circ}$ to the beam axis. The results in Figure $23(b)$ indicate that introducing a small angle makes the deformation behavior, and hence the identification, strongly sensitive to the in-plane shear modulus $\mathrm{G}_{12}$. A good convergence accuracy of $\mathrm{G}_{12}$ is seen up to $10 \%$ noise level.

Similar experiments have been performed with a beam subjected to a force couple acting about the beam axis, Figure 24, resulting in a twist deformation. This time the deformation is strongly dependent on $\mathrm{G}_{12}$ which is seen to converge well for all noise levels as shown in Figure 25. However, the convergence of $E_{1}$ is the poorest of all cases considered in this work.

The study of beam deformation with reference to the identifiability of specific material parameters shows that finding all parameters with this type of arrangement is very difficult. Moreover, this arrangement does not offer the convenience of altering the heterogeneity of the displacement field 
apart from changing the aspect ratio and fiber orientation. The plate specimen, fixed at three points, in comparison offers much more control parameters like aspect ratio, position of supports (along the four edges of plate) and load application point as well as the fiber orientation. A plate clamped at the edges and bent under pressure would not be able to offer such flexibility even though an analytical solution would be available to assist and speed up the identification.

\section{References}

1. Avril S, Bonnet M, Bretelle AS et al (2008) Overview of Identification Methods of Mechanical Parameters Based on Full-field Measurements. Exp Mech 48(4):381-402.

2. Grediac M, Hild F (2012) Full-Field Measurements and Identification in Solid Mechanics. Wiley, London.

3. Pierron F and Grediac M (2012) The Virtual Fields Method. Springer, New York.

4. Syed-Muhammad K, Toussaint E, and Grediac M (2009) Optimization of a mechanical test on composite plates with the virtual fields method. Struct Multidisc Optim 38:71-82.

5. Hild F, Roux S (2006) Digital image correlation: from displacement measurement to identification of elastic properties - a review. Strain 42:69-80.

6. M. Z. Siddiqui \& M. F. Ahmed (2014) An Out-of-Plane Motion Compensation Strategy for Improving Material Parameter Estimation Accuracy with 2D Field Measurements. Exp Mech 54(7):1259-1268.

7. Silva GHC (2009) Identification of Material Properties Using Finite Elements and Full-Field Measurements with Focus on the Characterization of Deterministic Experimental Errors. PhD thesis, Université Jean Monnet, France.

8. Behzad R (2014) In-Situ Mechanical Properties Identification of Composite Materials Using Inverse Methods Based on Full-Field Measurements. PhD thesis, Université De Montréal, Canada. 
9. Florentin $\mathrm{E}$, Lubineau $\mathrm{G}$ (2010) Identification of the parameters of an elastic material model using the constitutive equation gap method. Comp Mech 46(4):521-531.

10. Kashif SM (2007) Onto some numerical issues in the Virtual Fields Method: optimization of testing configuration and piecewise construction of virtual fields. PhD thesis, Université BLAISE PASCAL, France.

11. Avril S, Bonnet M, Bretelle AS et al (2008) Overview of Identification Methods of Mechanical Parameters Based on Full-field Measurements. Exp Mech 48(4):381-402.

12. Sutton MA, Orteu JJ, Schreier HW (2009) Image Correlation for Shape, Motion and Deformation Measurements. Springer, New York.

13. Chu TC, Ranson WF, Sutton MA, Peters WH (1985) Applications of Digital Image-Correlation Techniques to Experimental Mechanics. Exp Mech 25(3):232-244.

14. Luo PF, Chao YJ, Sutton MA, Peters WH (1993) Accurate Measurement of Three-dimensional Deformations in Deformable and Rigid Bodies Using Computer Vision. Exp Mech 33(2):123132.

15. Sutton MA, Yan JH, Tiwari V, Schreier HW, Orteu JJ (2008) The Effect of Out-of-plane Motion on 2D and 3D Digital Image Correlation Measurements. Opt Lasers Eng 46(10):746-757.

16. Haddadi H, Belhabib S (2008) Use of Rigid-body Motion for the Investigation and Estimation of the Measurement Errors Related to Digital Image Correlation Technique. Opt Lasers Eng 46(2):185-196.

17. Siddiqui MZ (2015) Towards Eliminating the Displacement Bias Due to Out-of-plane Motion in 2D Inverse Problems: A Case of General Rigid-Body Motion. Strain 51:55-70.

18. Timoshenko SP, Woinowsky-Krieger S (1959) Theory of plates and shells, $2^{\text {nd }}$ ed. McGrawHill, New York.

19. Mbakogu FC, Pavlovic MN (2000) Bending of clamped orthotropic rectangular plates: A variational symbolic solution. Comp Struct 77:117-128. 
20. TAIRYFIL TC-36S datasheet, Sage Zander Ltd. United Kingdom.

21. SWANCOR 2552 epoxy resin datasheet, SWANCOR Inc. Co., Ltd. Taiwan.

22. Siddiqui MZ, Tariq F, Naz N (2011) Application of a two-step digital image correlation algorithm in determining Poisson's ratio of metals and composites. International Astronautical Federation. http://www.iafastro.net/iac/archive/browse/IAC-11/C2/8/10236. Accessed 31 $31^{\text {st }}$ November 2016.

23. DIC Challenge - Society for Experimental Mechanics. https://sem.org/dic-challenge. Accessed $7^{\text {th }}$ December 2016.

24. Test Methods for Constituent Content of Composite Materials. ASTM standard (2004) D3171-99R04.

25. Mechanical Properties of Carbon Fiber Composite Materials, Fiber/Epoxy resin $\left(120^{\circ} \mathrm{C}\right.$ Cure). Performance Composites Ltd. http://www.performance-composites.com/carbonfibre/ mechanicalproperties 2.asp. Accessed 29th January 2017.

26. Bednarcyk BA, Aboudi J, Arnold SM (2014) The effect of general statistical fiber misalignment on predicted damage initiation in composites. Composites Part B: Engineering, 66:97-108

27. Ncorr analysis of DIC challenge datasets. Available as supplementary material on https://link.springer.com/article/10.1007/s11340-015-0009-1. Accessed 7 $7^{\text {th }}$ December 2016.

28. Blaber J, Adair B, Antoniou A (2015) Ncorr: Open-Source 2D Digital Image Correlation Matlab Software. Experimental Mechanics, 55(6):1105-1122.

29. Correlated Solutions - VIC-2DTM. https://correlatedsolutions.com/vic-2d/. Accessed $7^{\text {th }}$ December 2016.

30. Siddiqui MZ (2016) 2D-DIC results with OSM for DIC challenge datasets provided by Society for Experimental Mechanics. https://www.researchgate.net/publication/311454837. Accessed 7th December 2016. DOI: 10.13140/RG.2.2.17655.83362. 


\section{Figures}

Figure 1 Solid geometry for the inverse problem

Figure 2 Effect of out-of-plane motion of a point on its perceived image

Figure 3 Coordiante system for pinhole camera system

Figure 4 Flowchart for the minimization problem

Figure 5 Centerlines and speckle pattern for assisting alignment and correlation during image acquisition

Figure 6 Pointed bolts at the support locations

Figure 7 Schematic of the specimen geometry, supports and load application points

Figure 8(a) Front view of test plate and (b) Back of the plate with load applicatoin

Figure 9 Examination of composite specimen with optical microscope - 200x magnification

Figure 10 Fiber orientation analysis with optical microscope $-100 x$ magnification

Figure 11 Orientation of fibers identified by edge detection in all 14 images

Figure 12 Probability distribution function for fiber orientation

Figure 13 Decrease in maximum relative difference in deflection for two plates having fibers oriented at $0^{\circ}$ and $\pm 2.3^{\circ}$ with increasing number of layers

Figure 14 Representation of the simulated displacement noise

Figure 15 Convergence trend for estimated parameters with simulated data having $3 \%$ noise - Case I. Dashed lines represent target values. The curves show results with nine different random noise distributions.

Figure 16 Variation in estimated parameters as a function of simulated measurement noise - Case I

Figure 17 Variation in estimated parameters as a function of simulated measurement noise - Case II

Figure 18 Convergence trend for estimated parameters with experimental data at different loads Case I. Dashed lines represent target values

Figure 19 Convergence trend for estimated parameters with experimental data at different loads Case II. Dashed lines represent target values

Figure 20 OSM results for Sample 1 of DIC Challenge datasets. (a) Average Displacement Error (b) Local Displacement Error

Figure 21 OSM results for Sample 2 of DIC Challenge datasets. (a) Average Displacement Error (b) Local Displacement Error 
Figure 22 Beam specimen under out-of-plane bending load

Figure 23 Variation in estimated parameters as a function of simulated measurement noise for beam specimen under bending load. (a) $0^{\circ}$ orientation (b) $5^{\circ}$ orientation

Figure 24 Beam specimen under the action of force couple creating twist about beam axis

Figure 25 Variation in estimated parameters as a function of simulated measurement noise for beam specimen under twisting load

\section{Tables}

Table 1 Direct and inverse problems

Table 2 Properties of Carbon Fiber Yarn, TAIRYFIL [20]

Table 3 Properties of SWANCOR 2552 Epoxy resin [21]

Table 4 Resin and fiber percentages in composite specimen

Table 5 Elastic moduli of composite specimen from direct methods

Table 6 Bounds on elastic constants for the optimization problem

Table 7 Applied loads, initial guess in cost function minimization and estimated parameter values Case I

Table 8 Applied loads, initial guess in cost function minimization and estimated parameter values Case II 


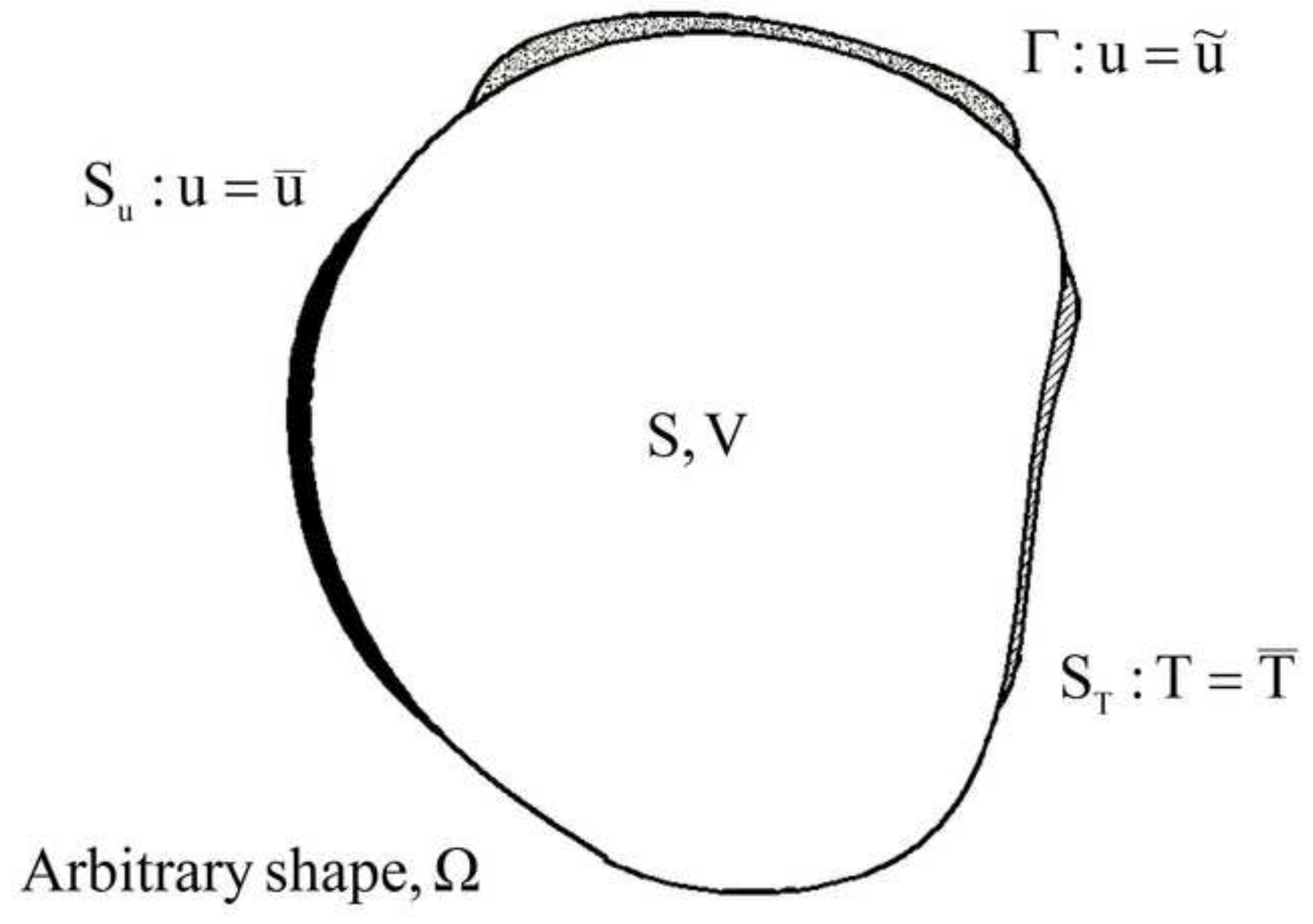




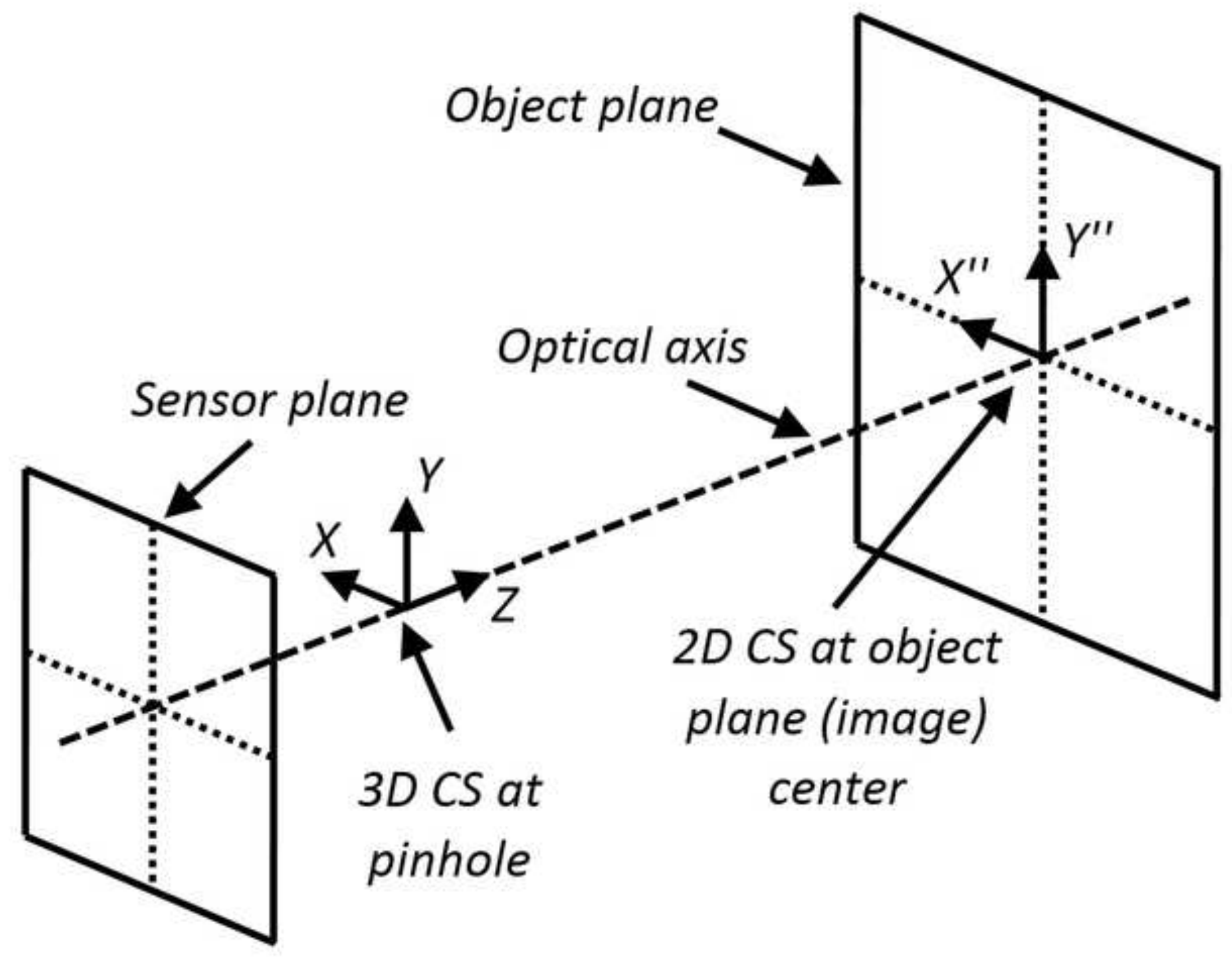


$\square$ Initial geometry

x Deformed geometry

- Projected geometry

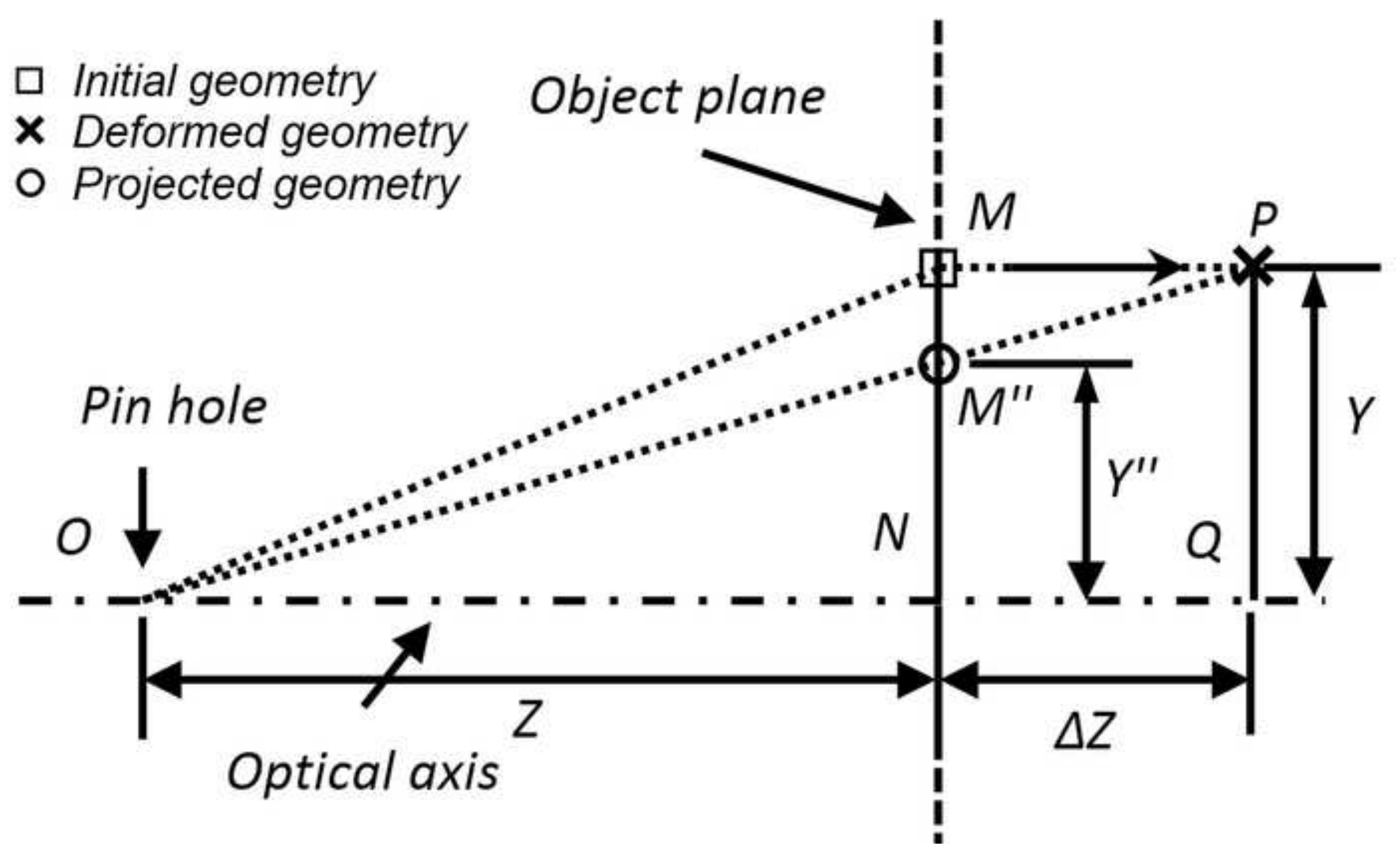

Pin hole

o $\downarrow$

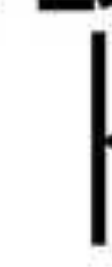

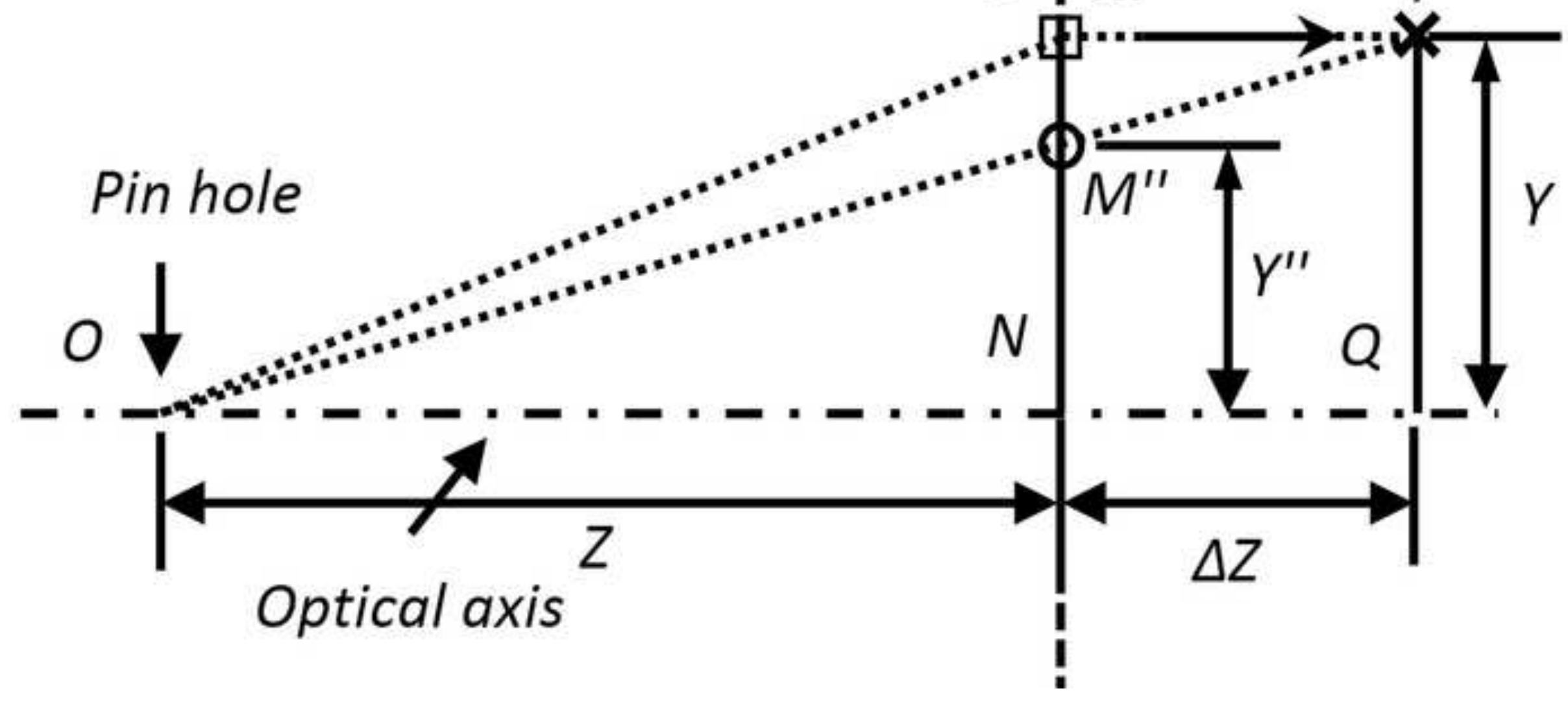




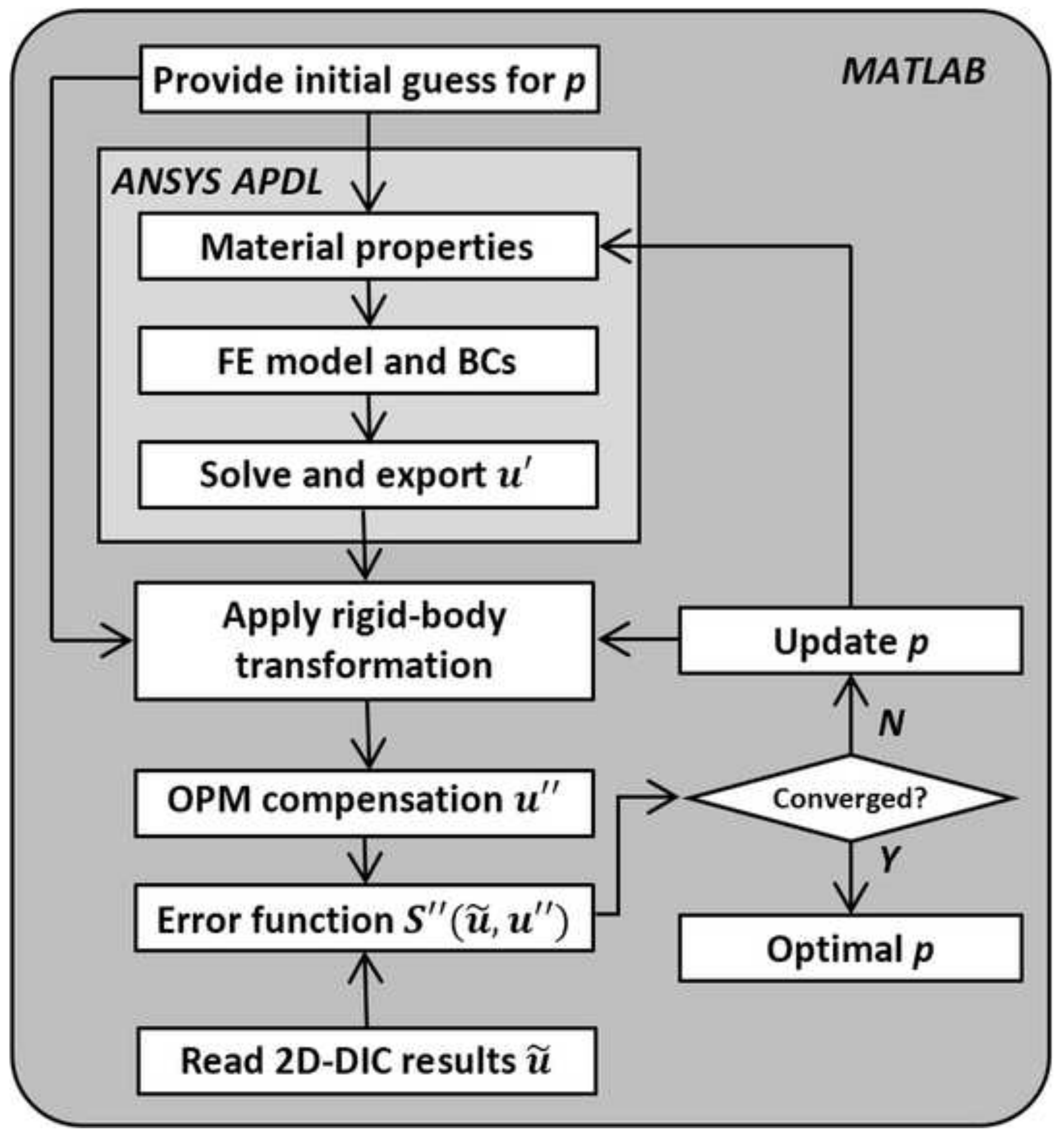




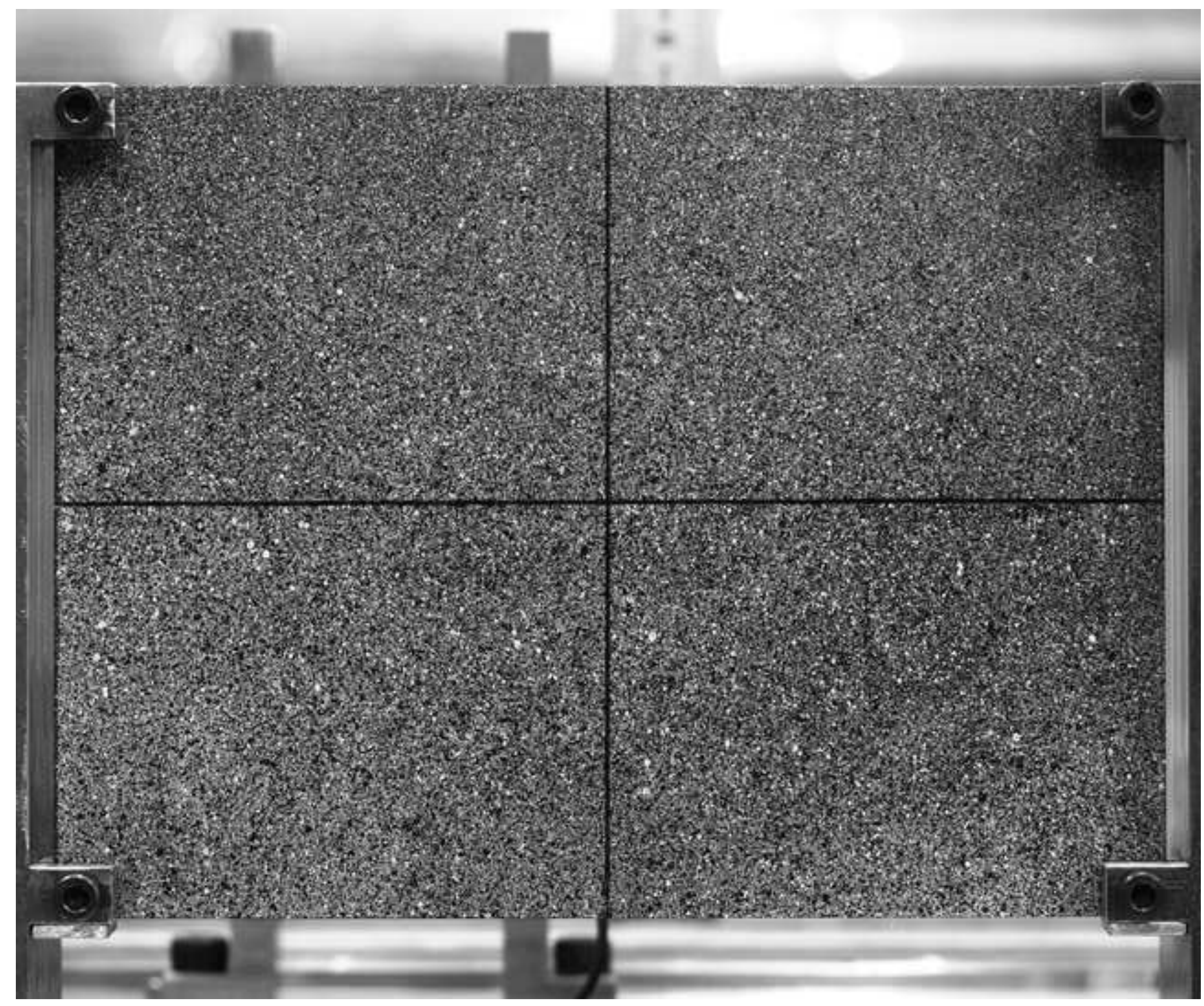




\section{Specimen holder}

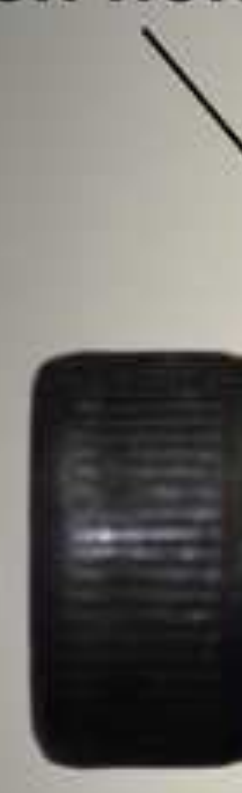

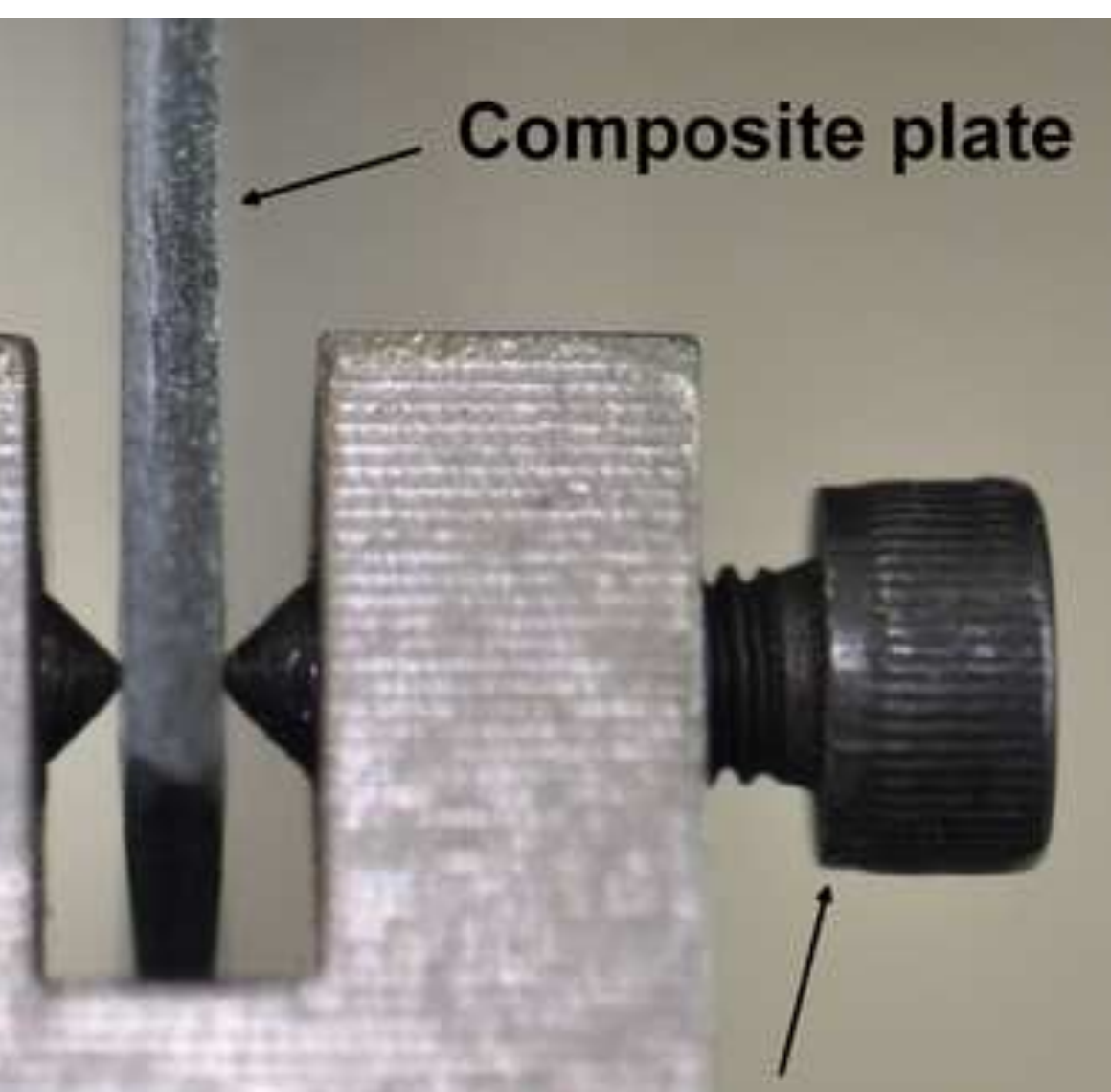

\section{Composite plate}

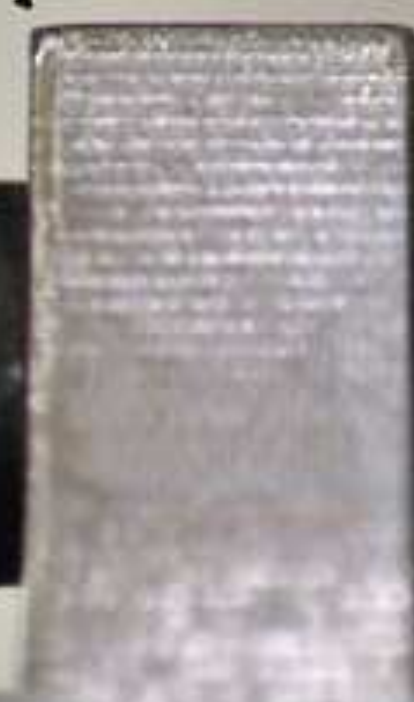

Pointed bolt 


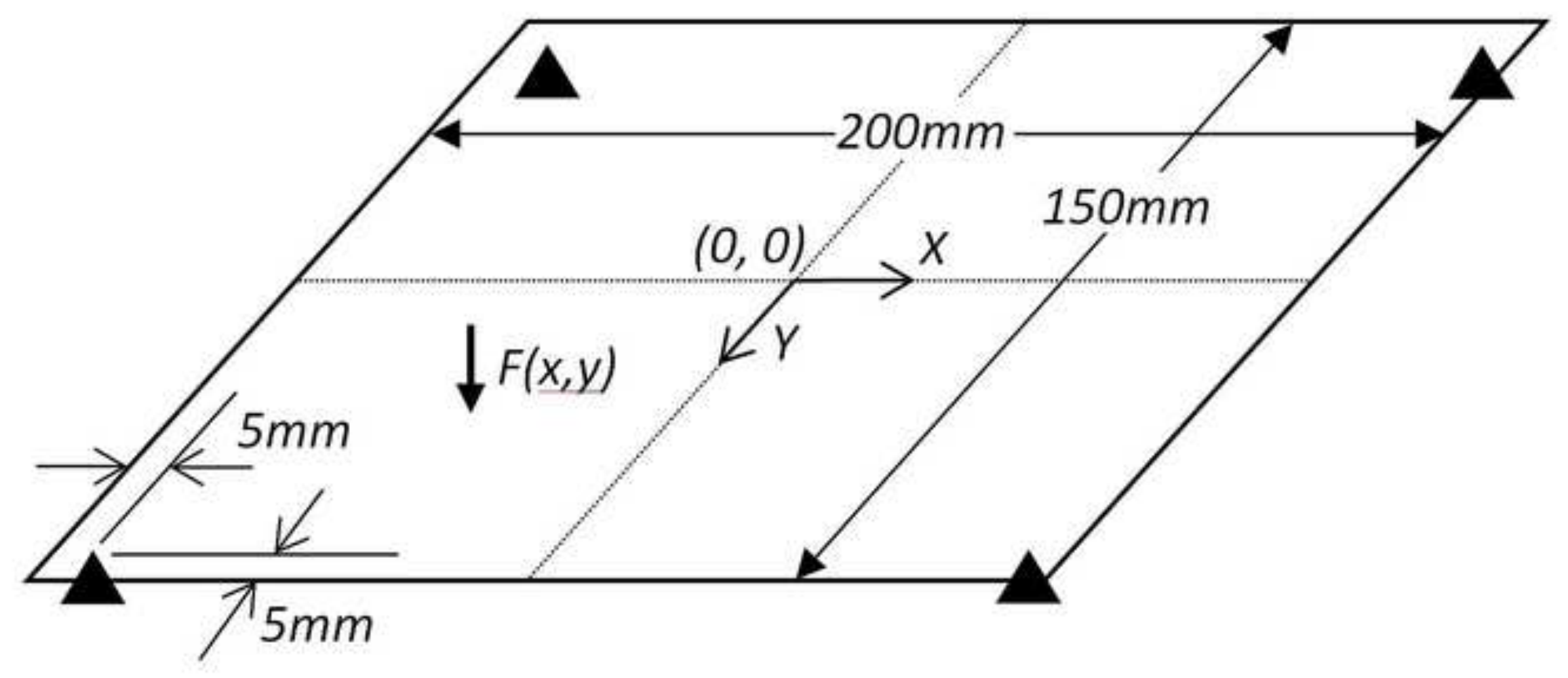




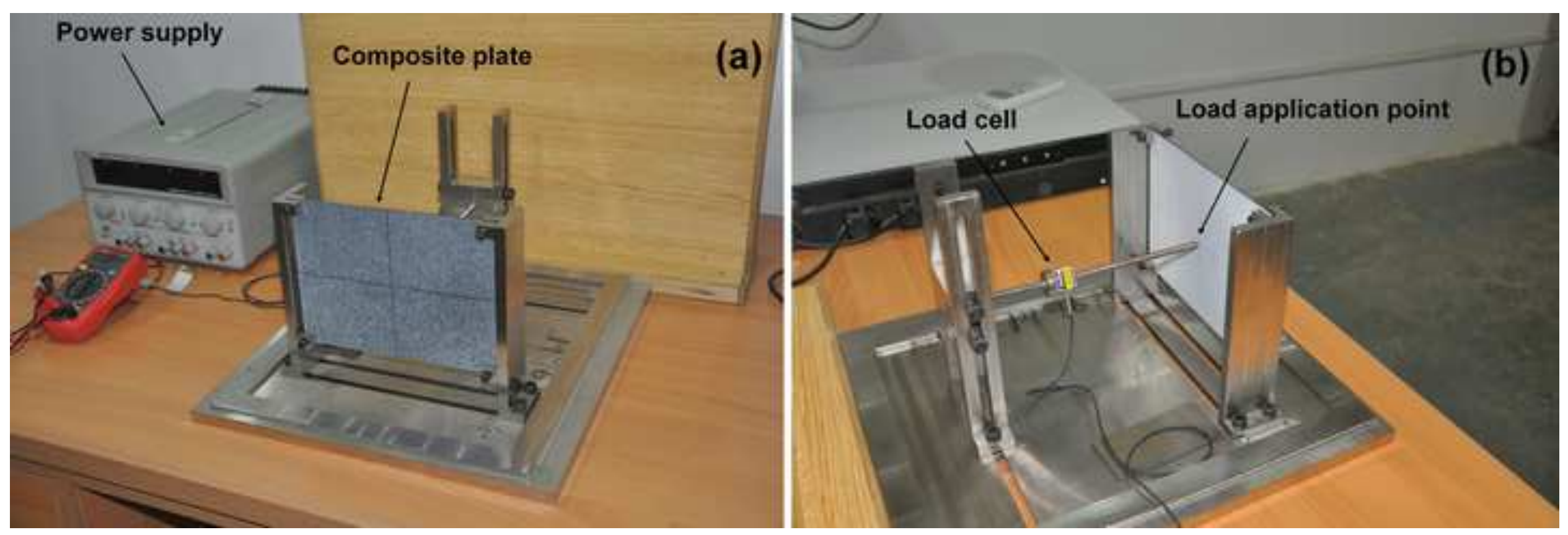




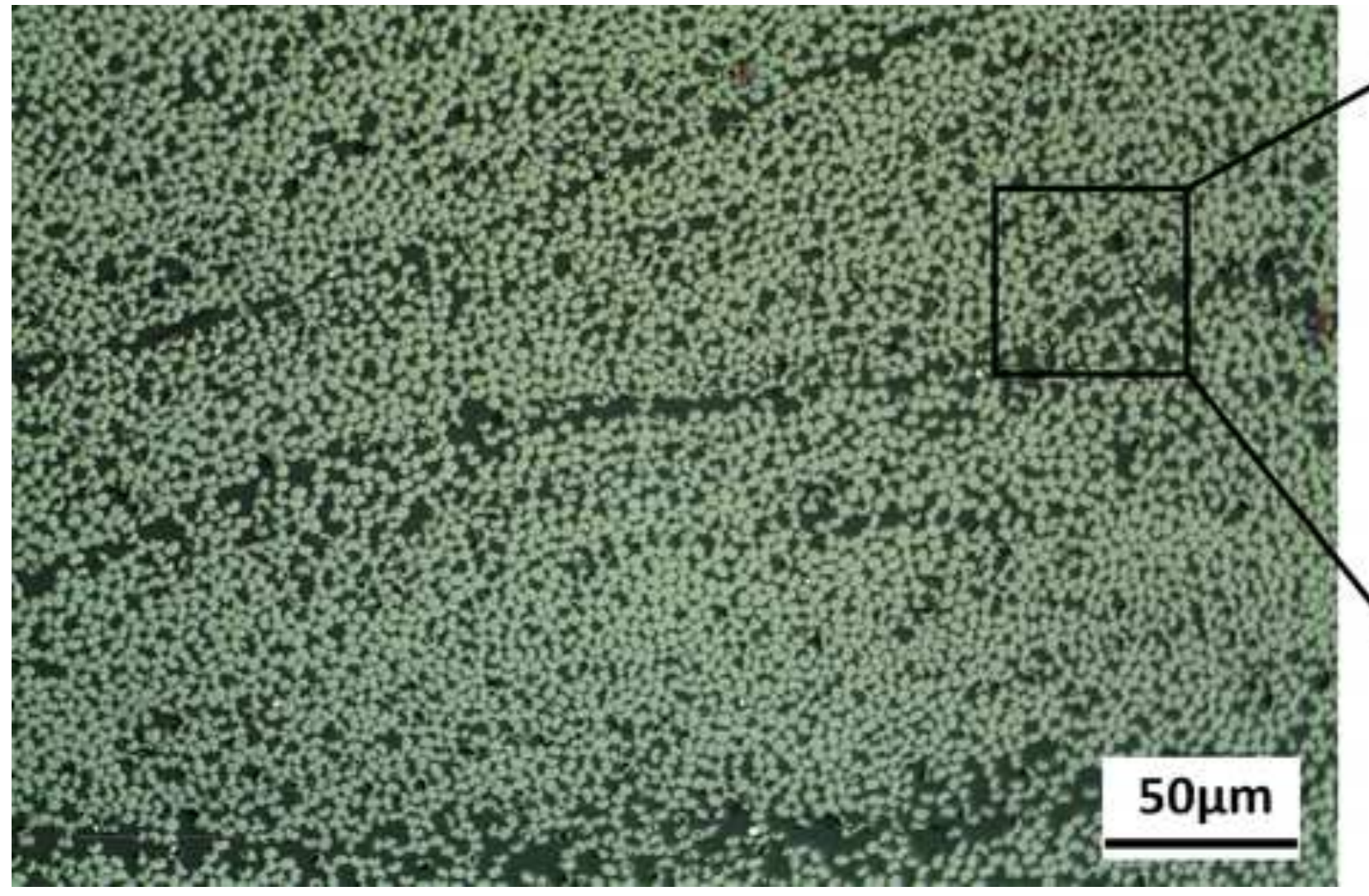

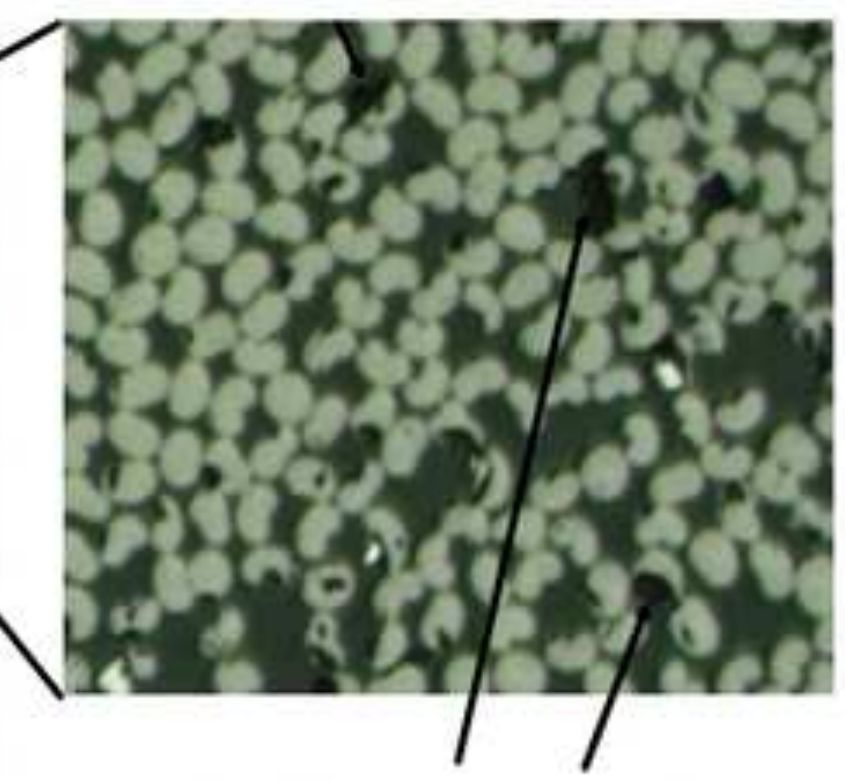

Small voids appearing as black spots 


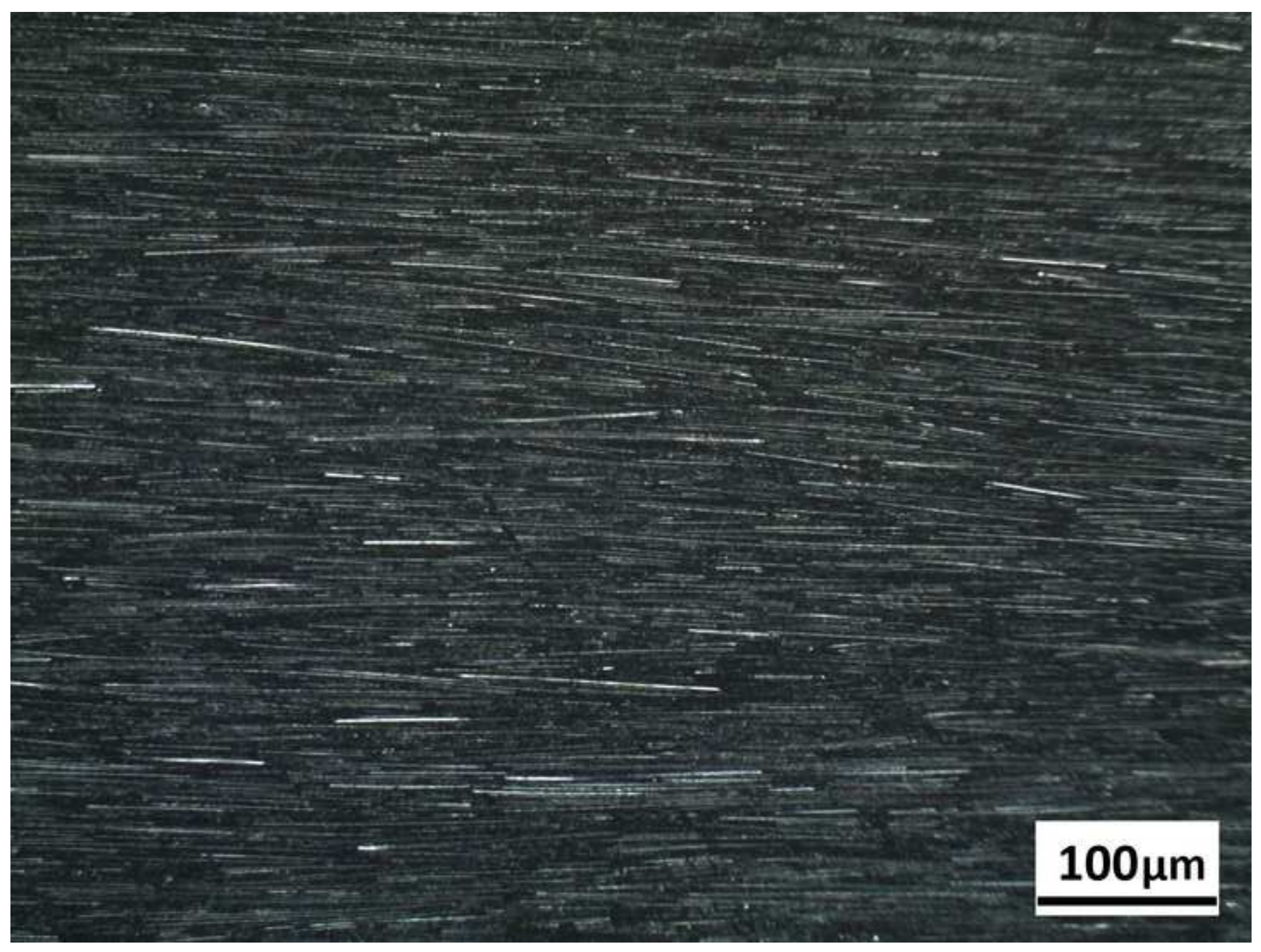




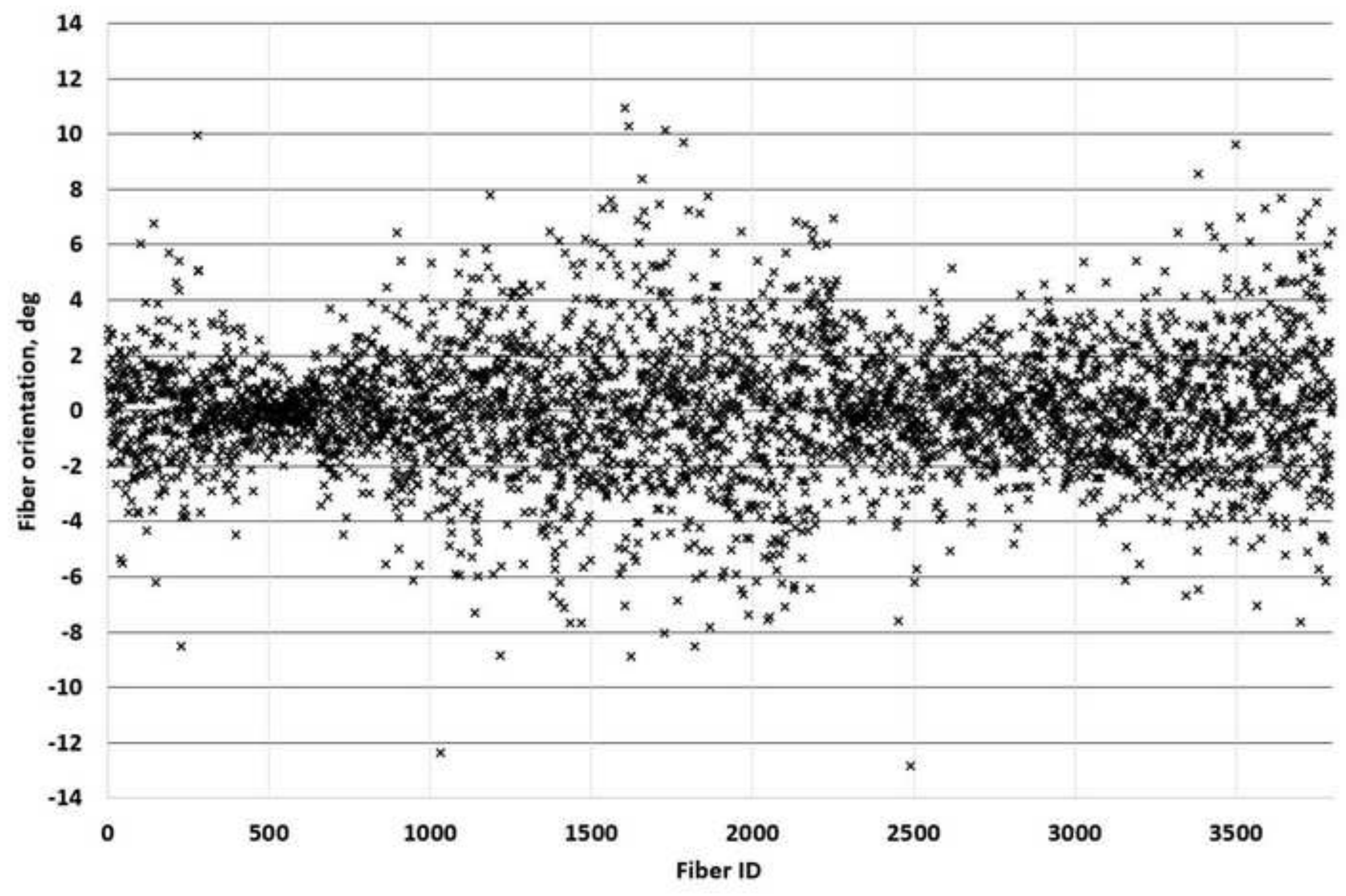




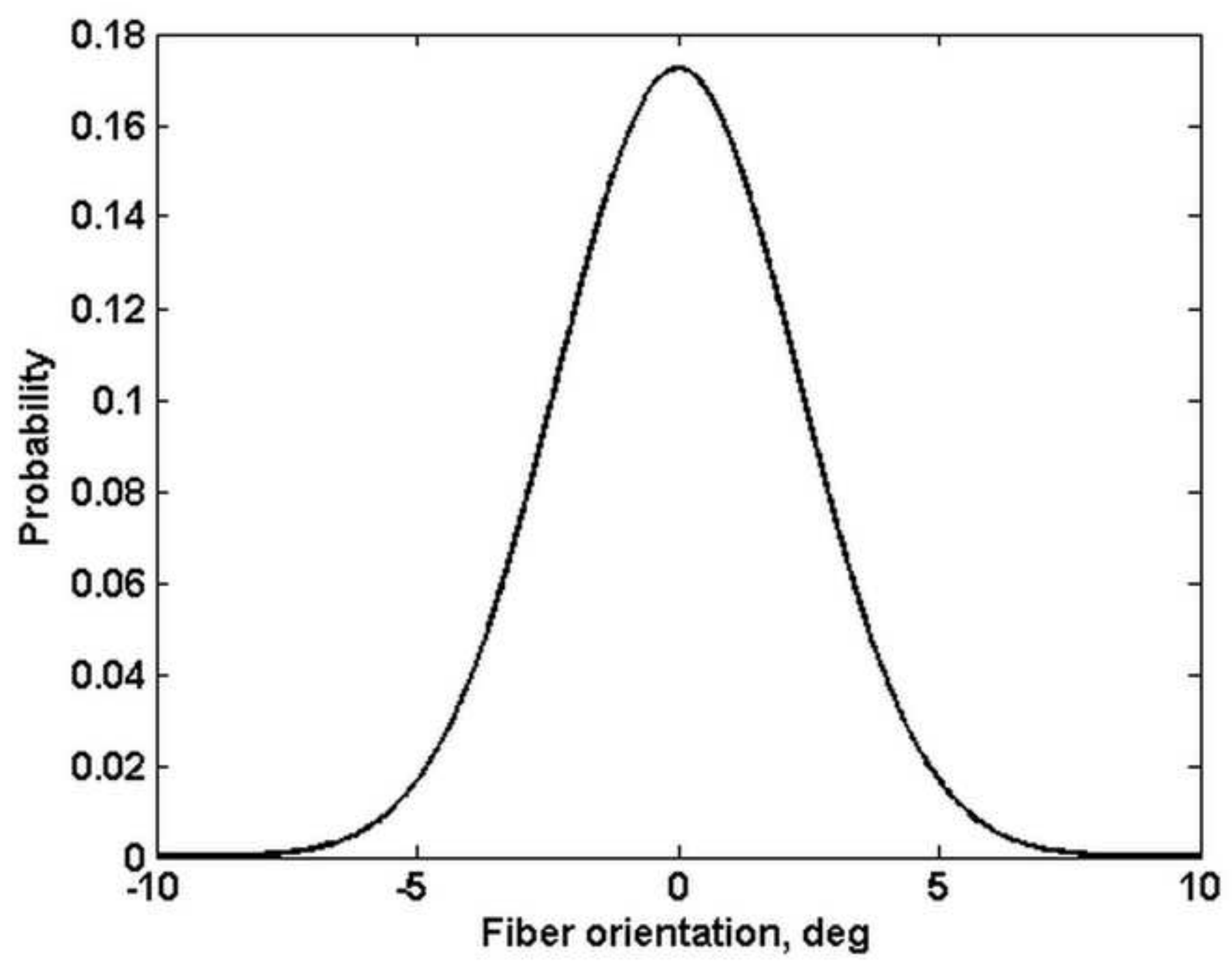




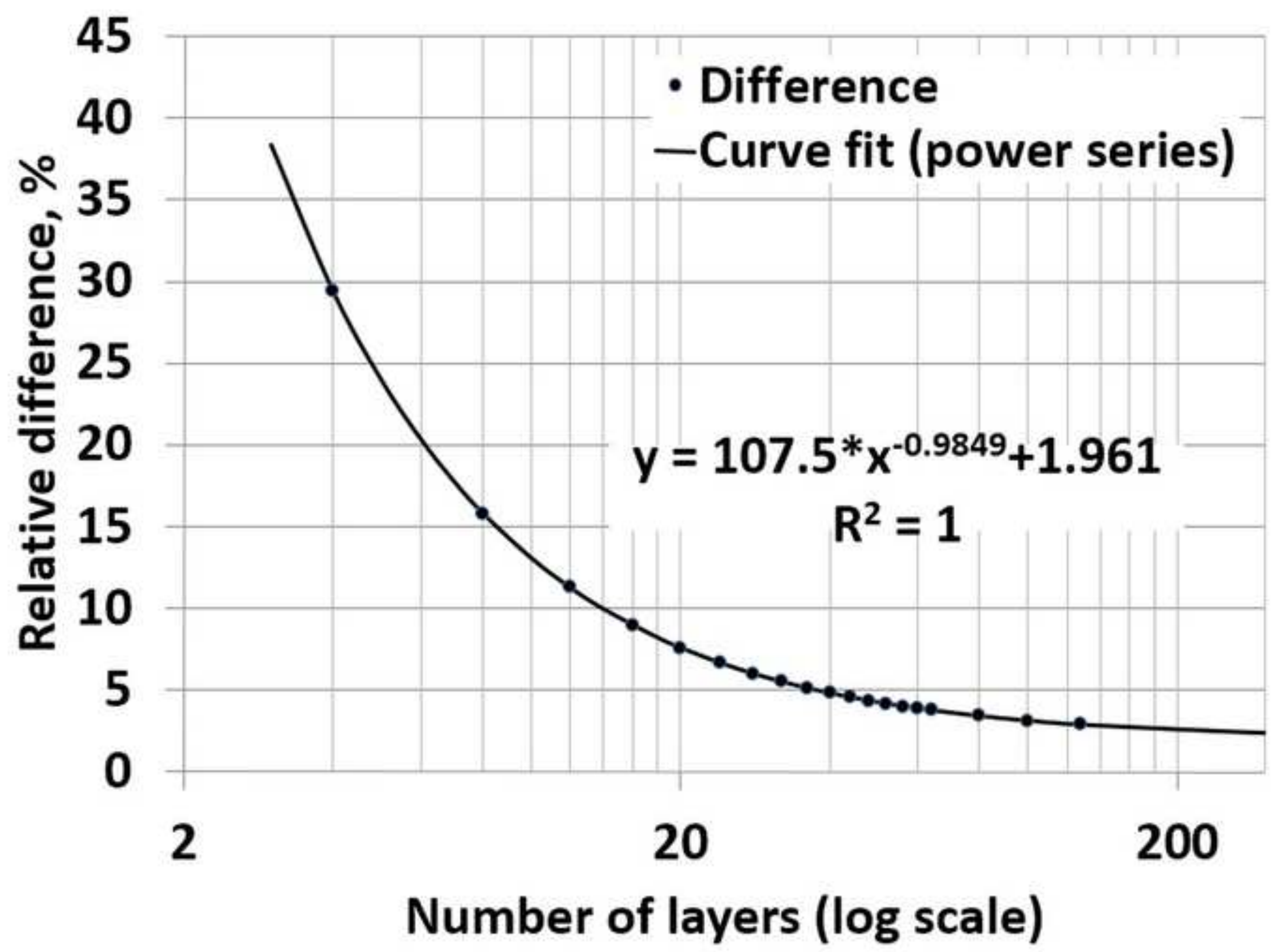




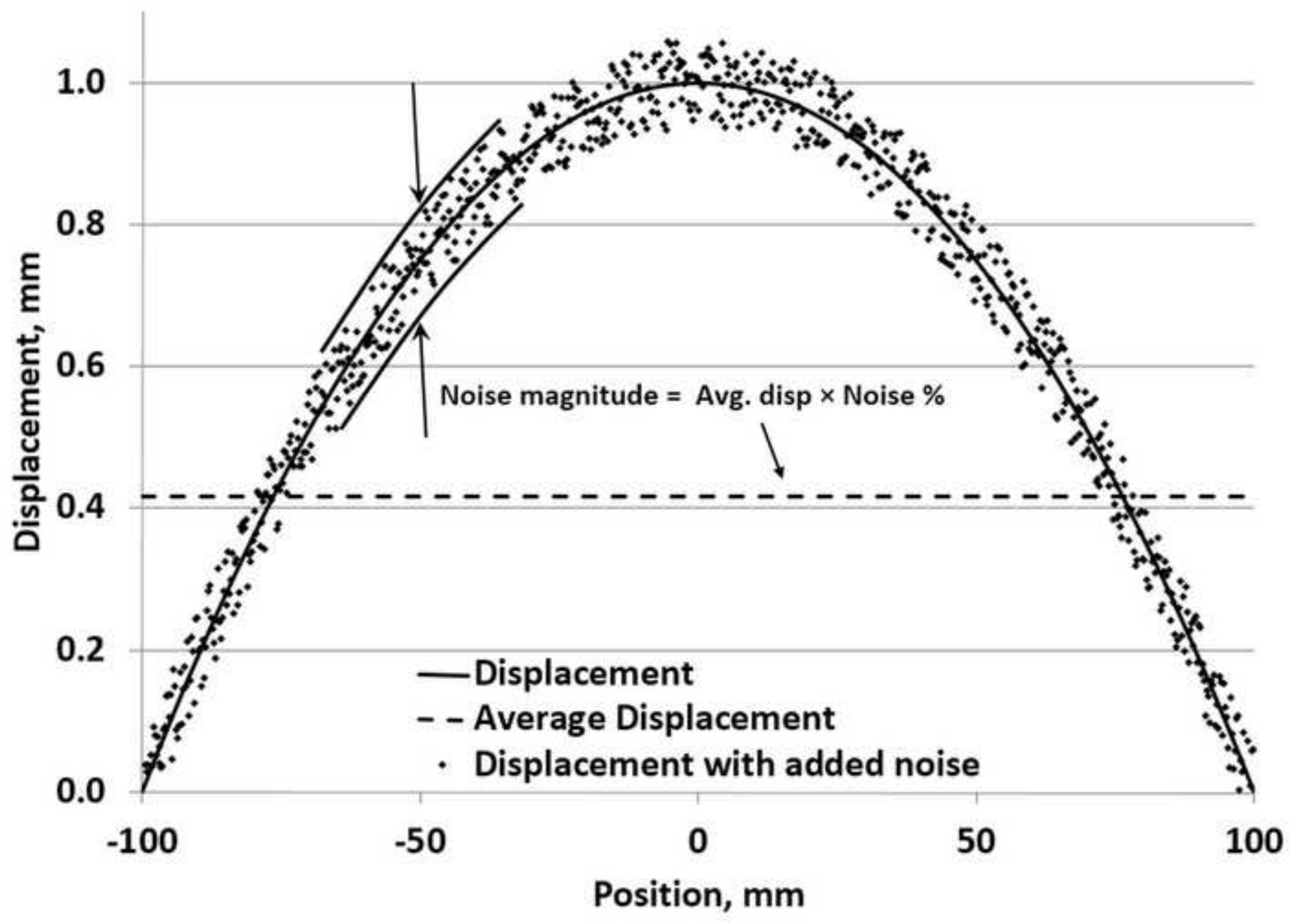



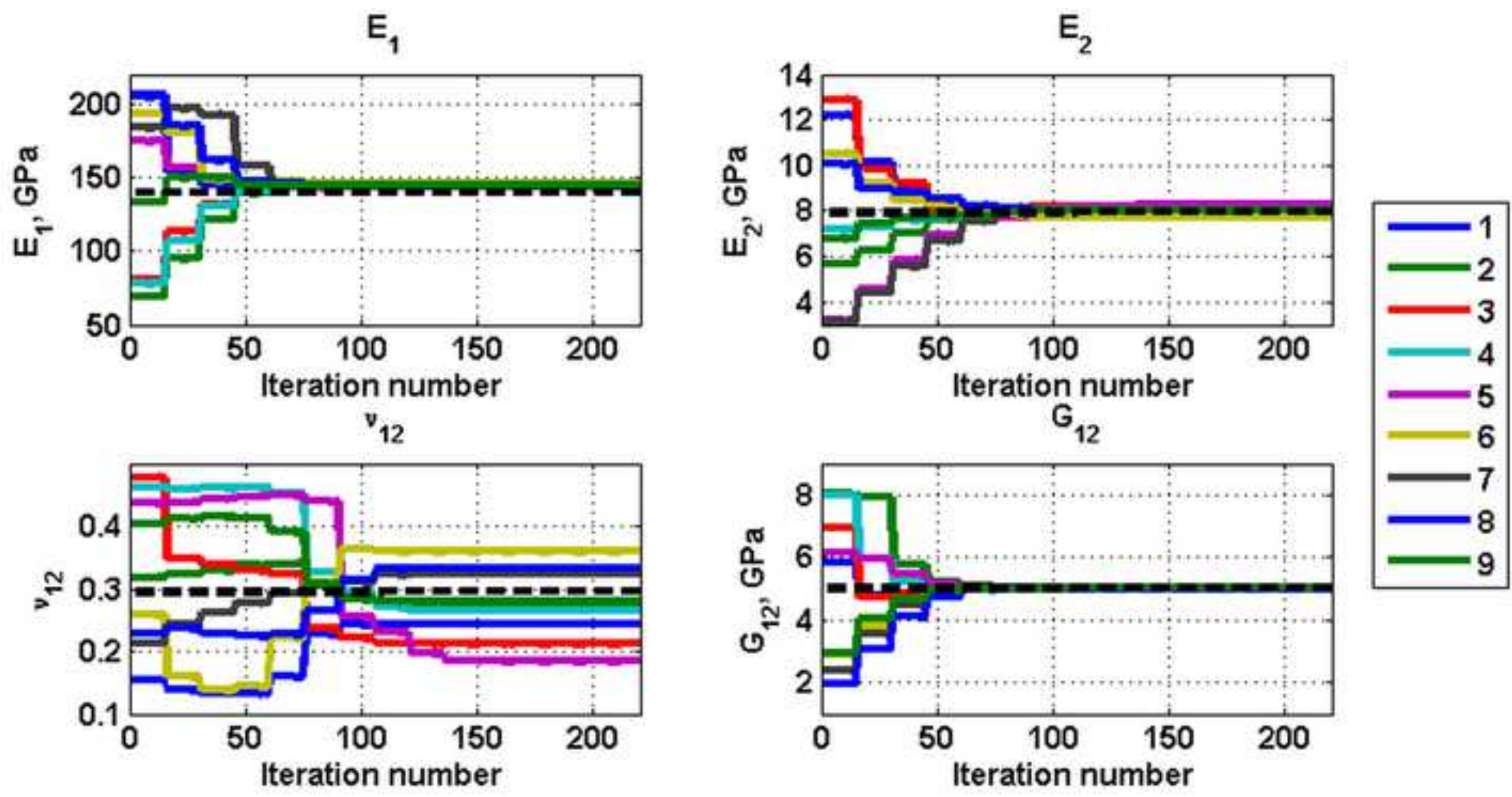


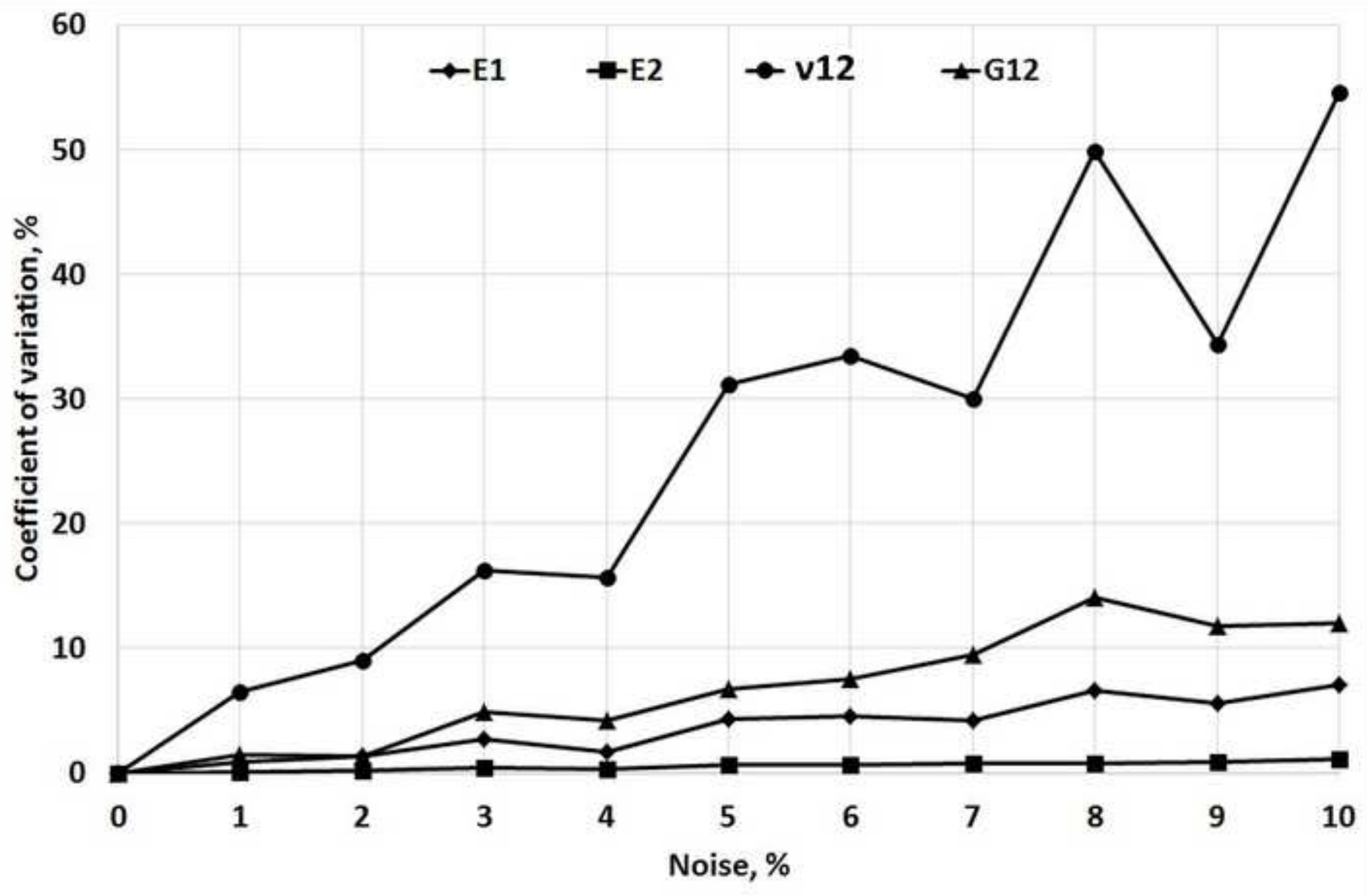




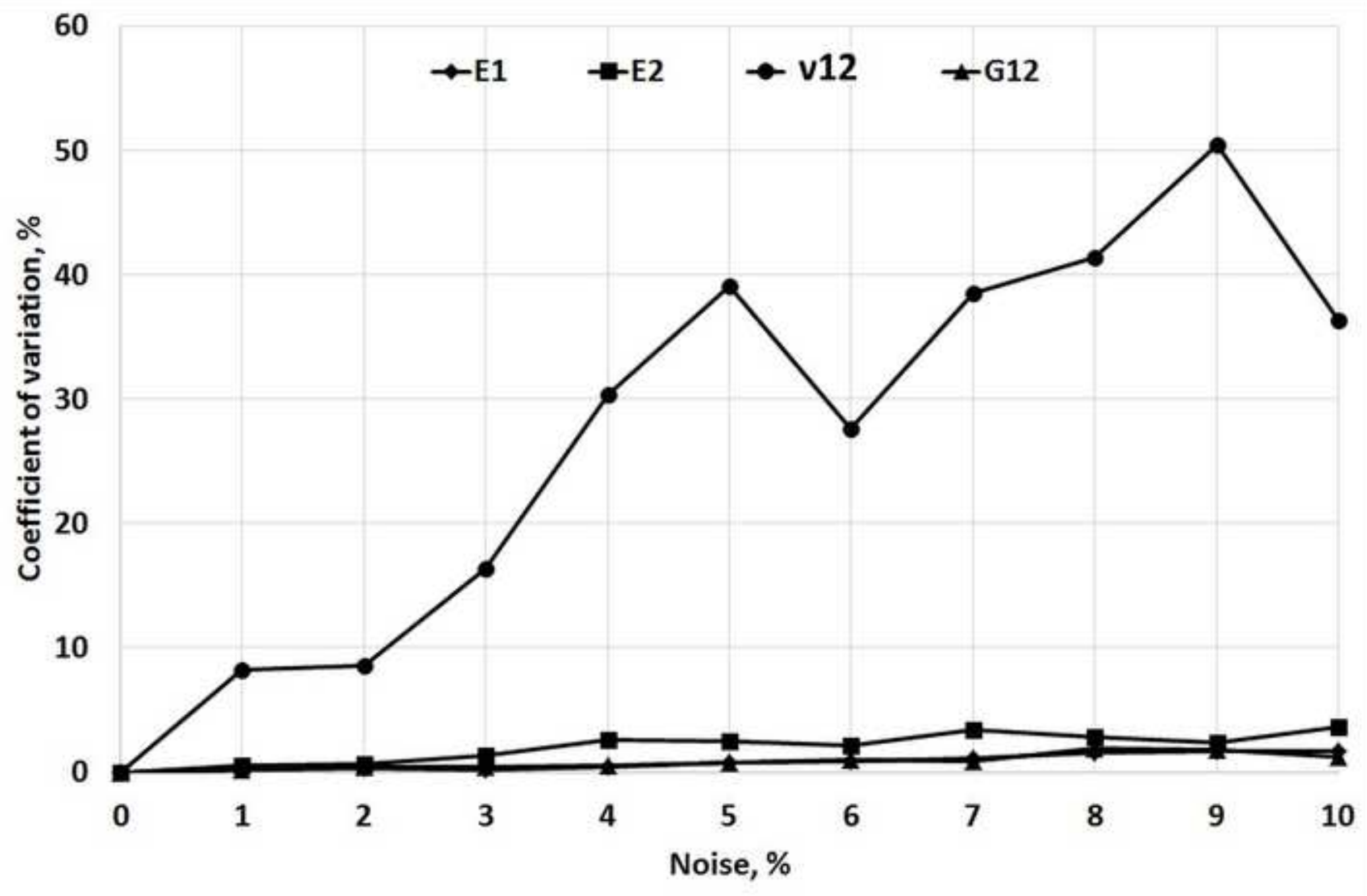



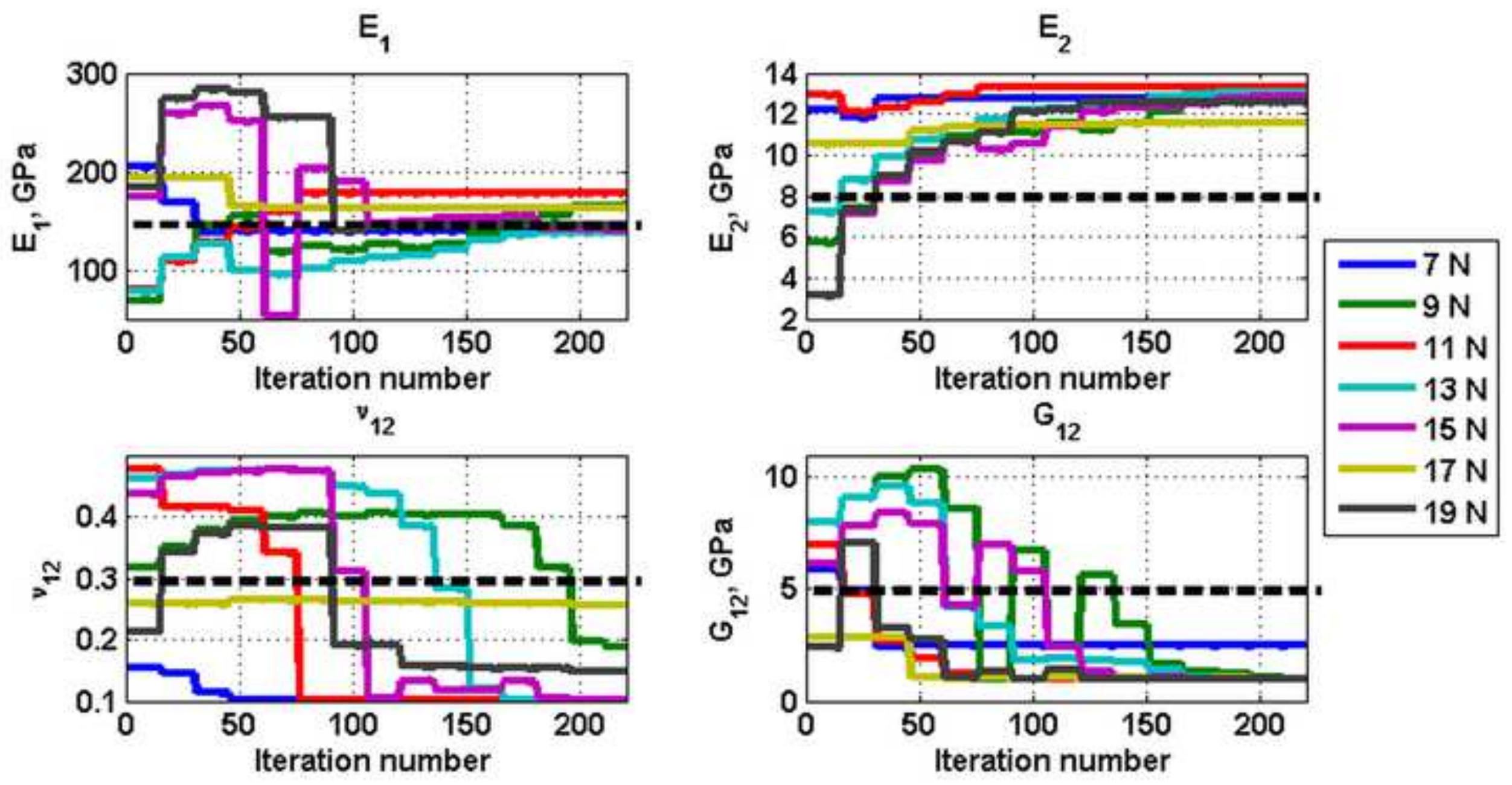

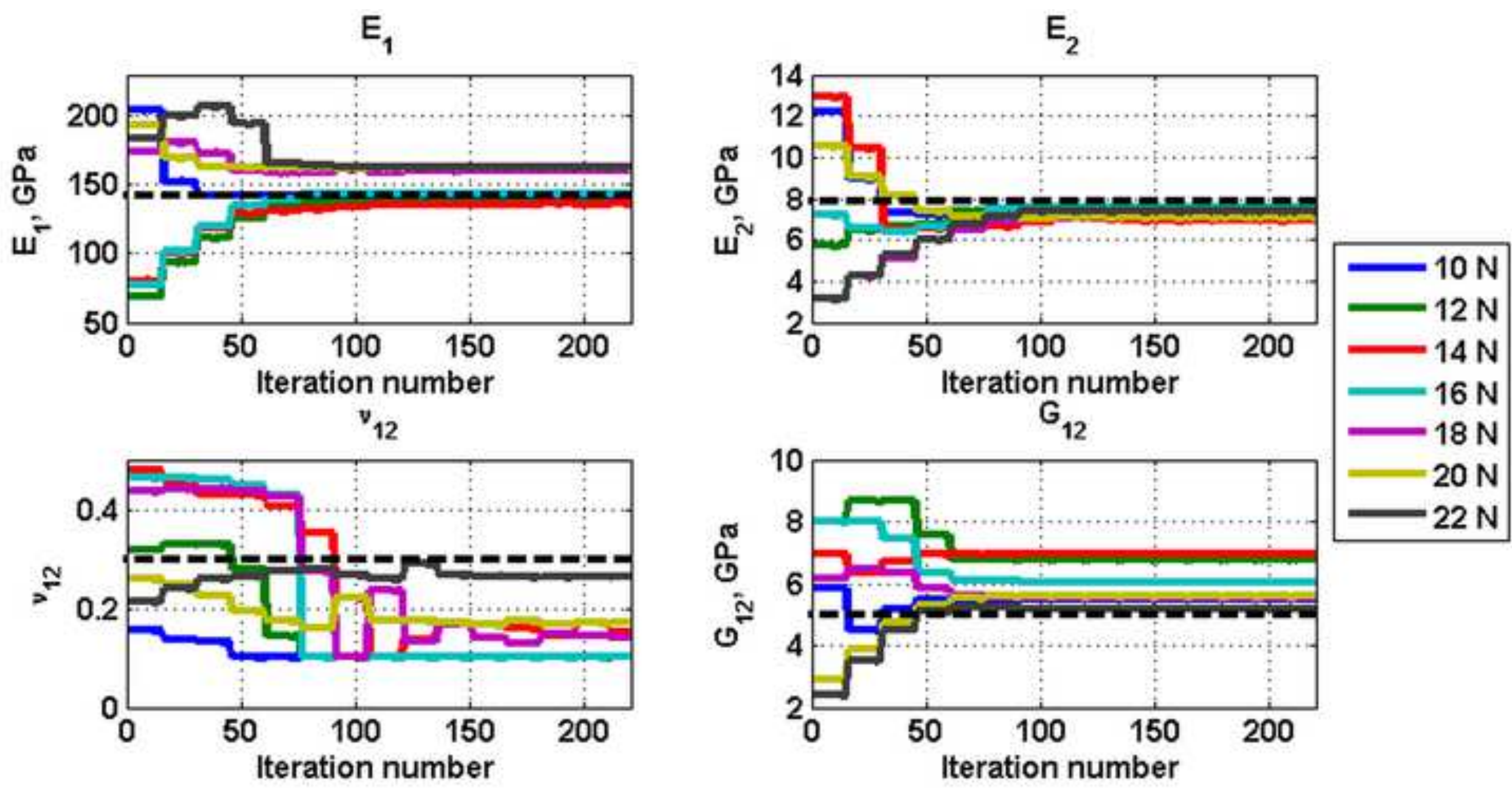


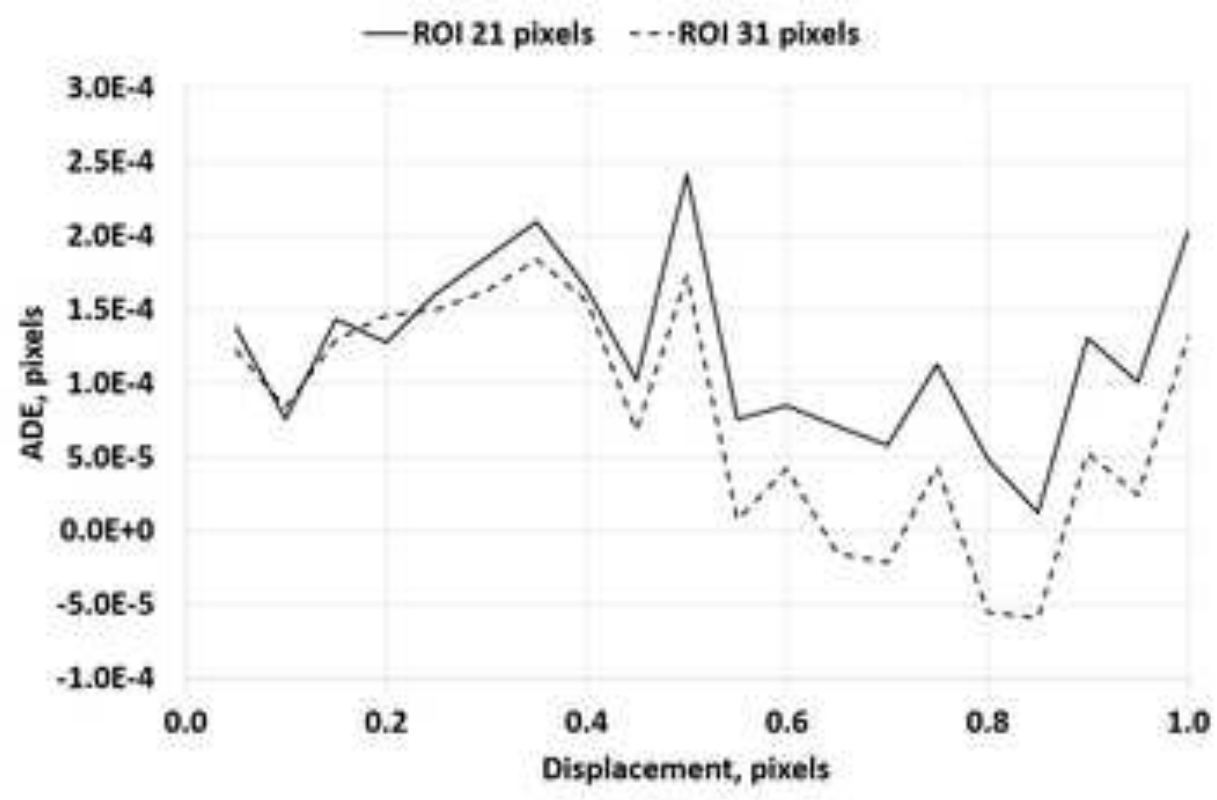

(a)

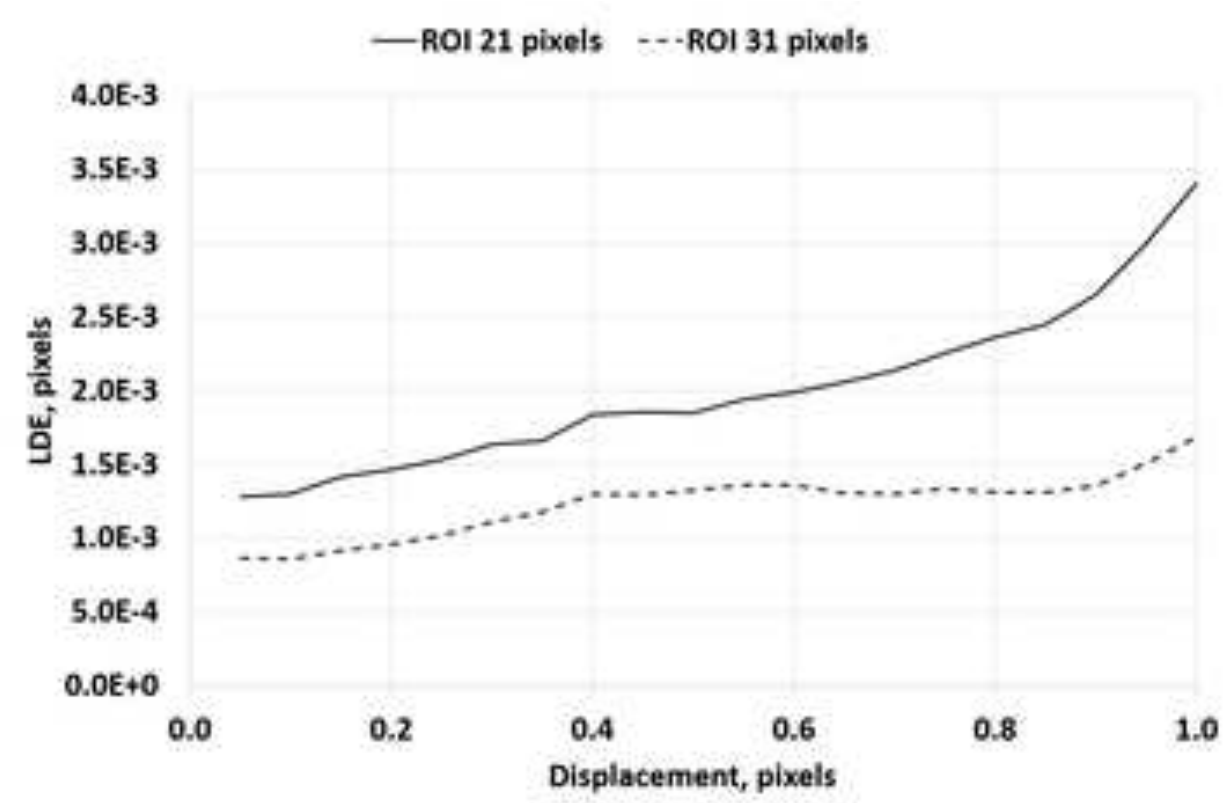

(b) 


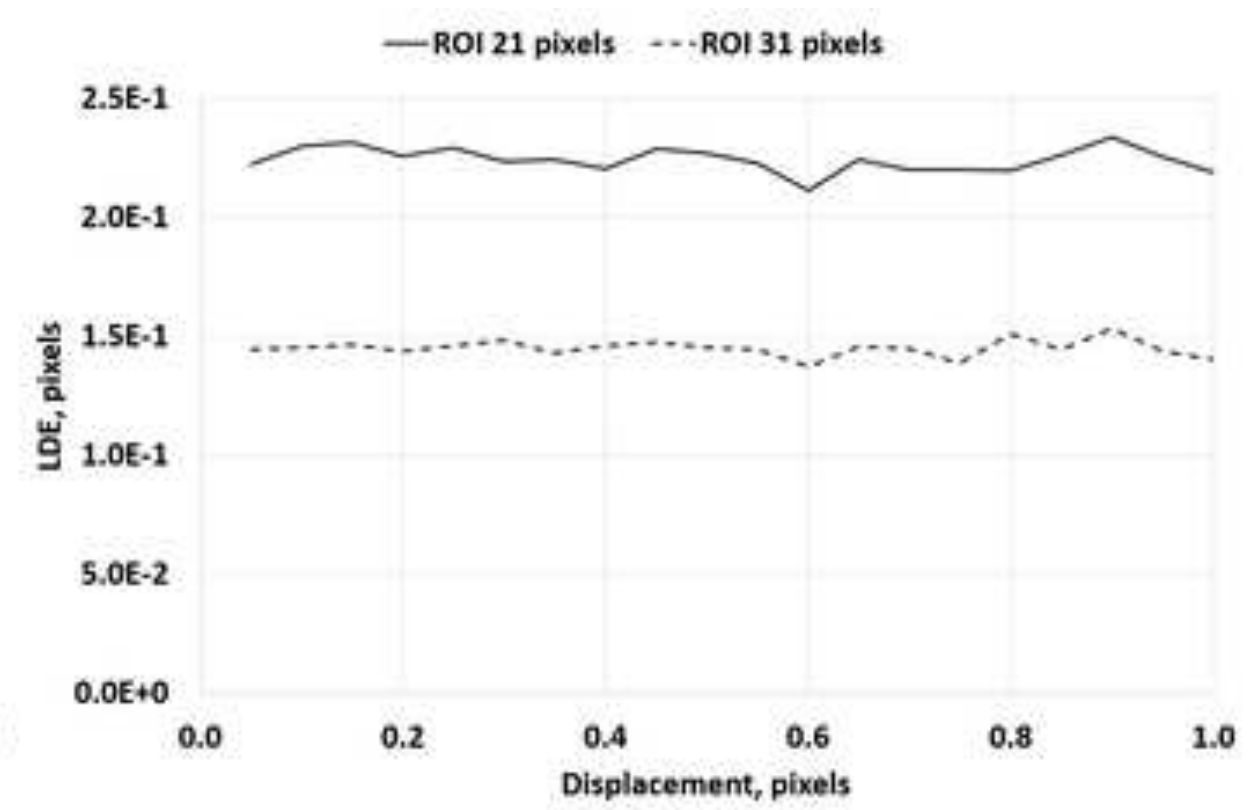

(b)

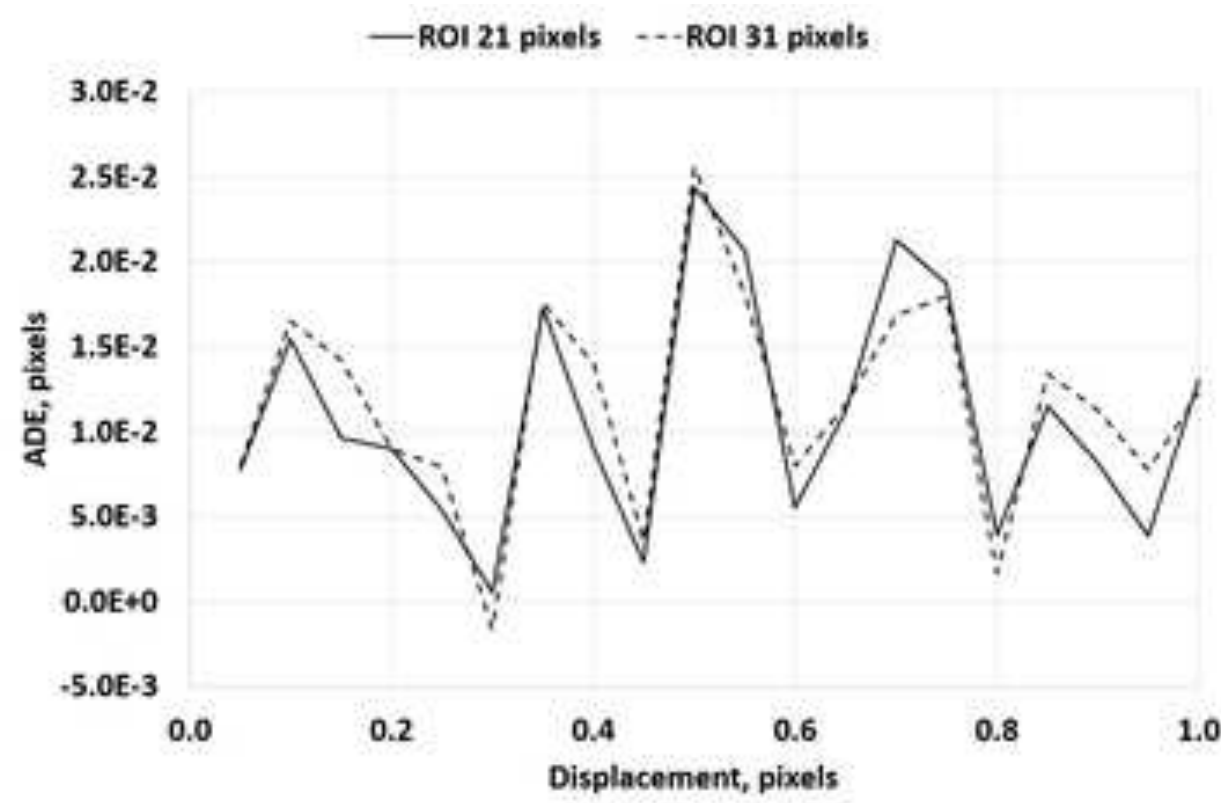

(a) 

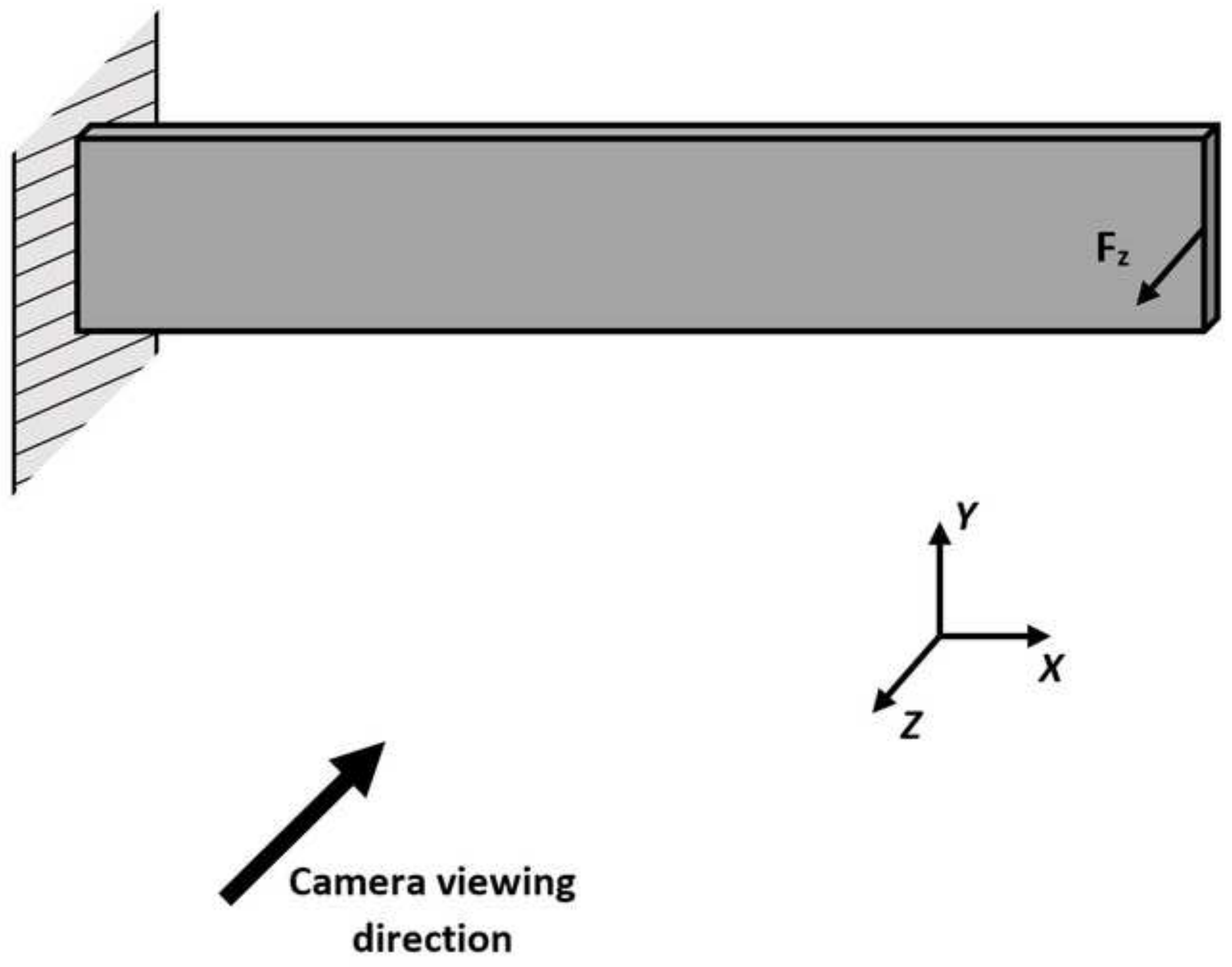


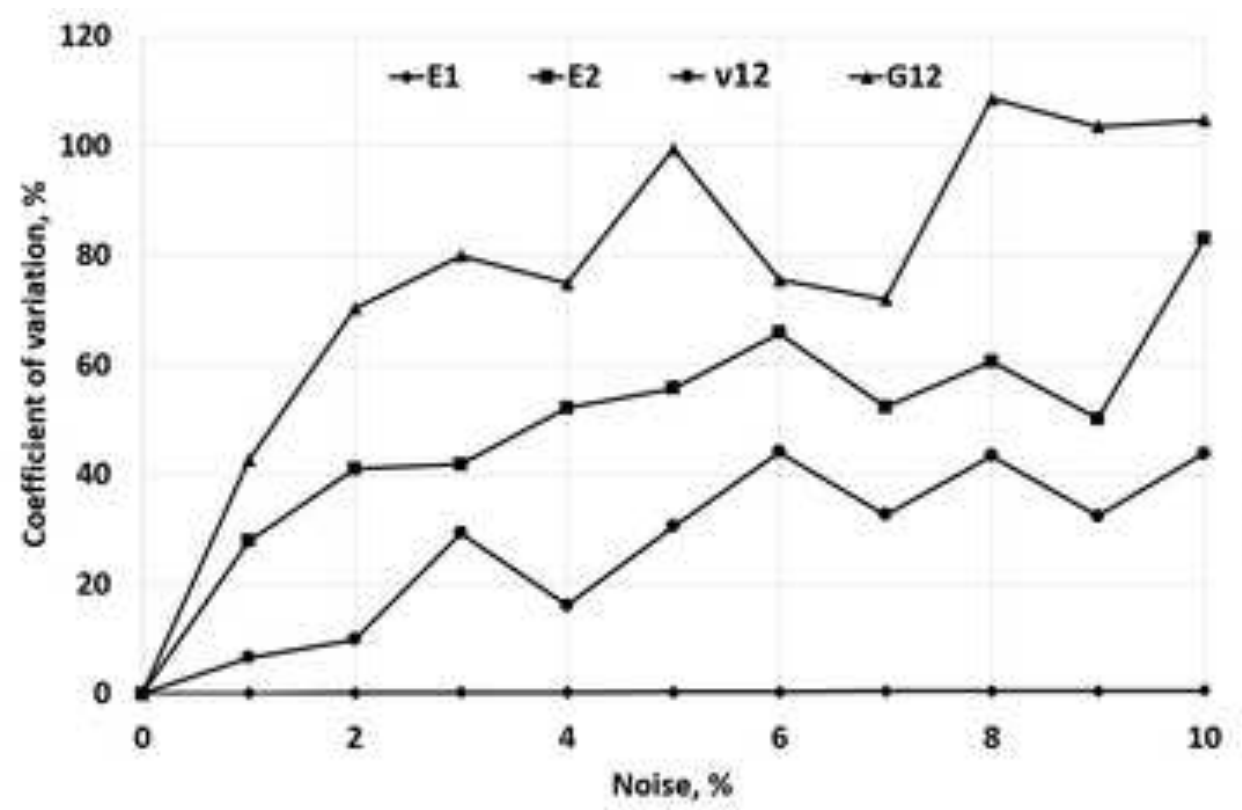

(a)

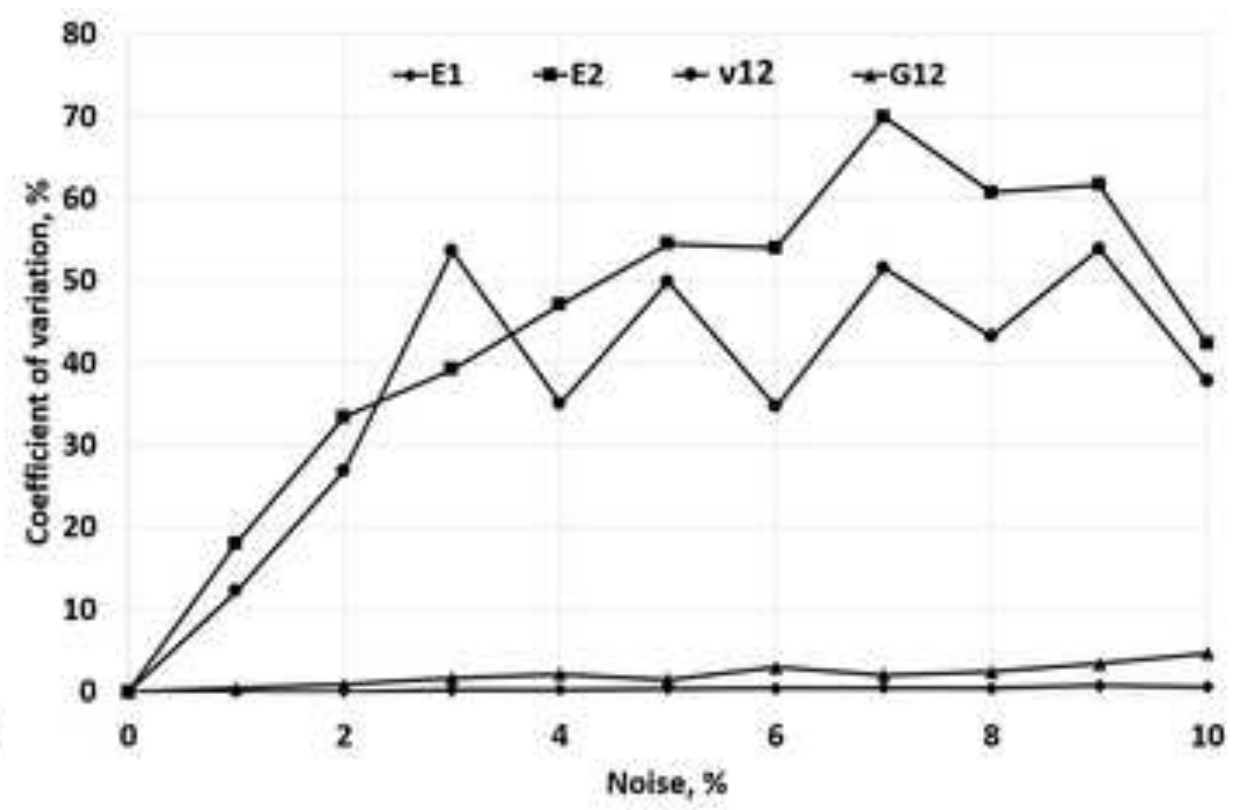

(b) 


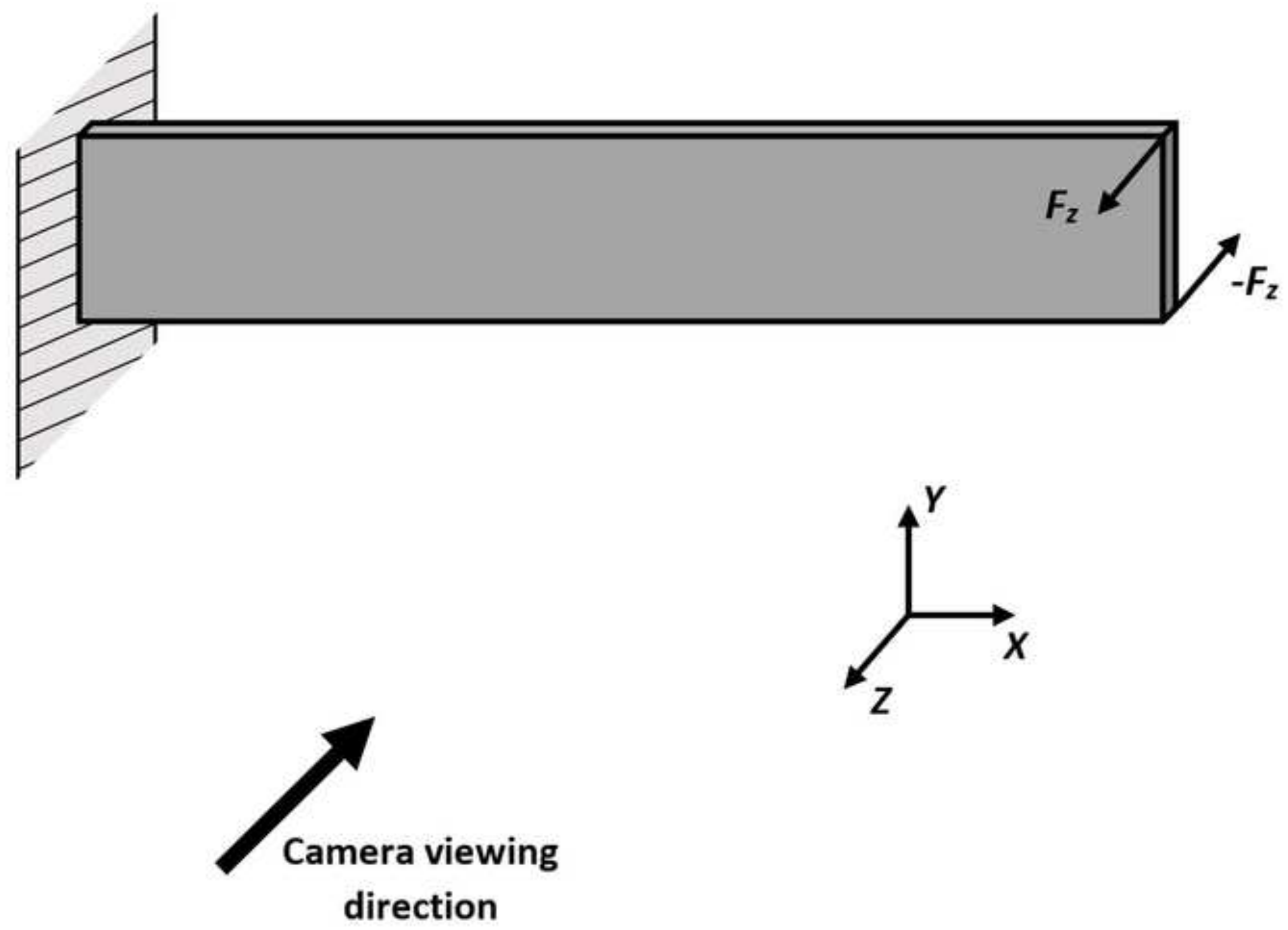




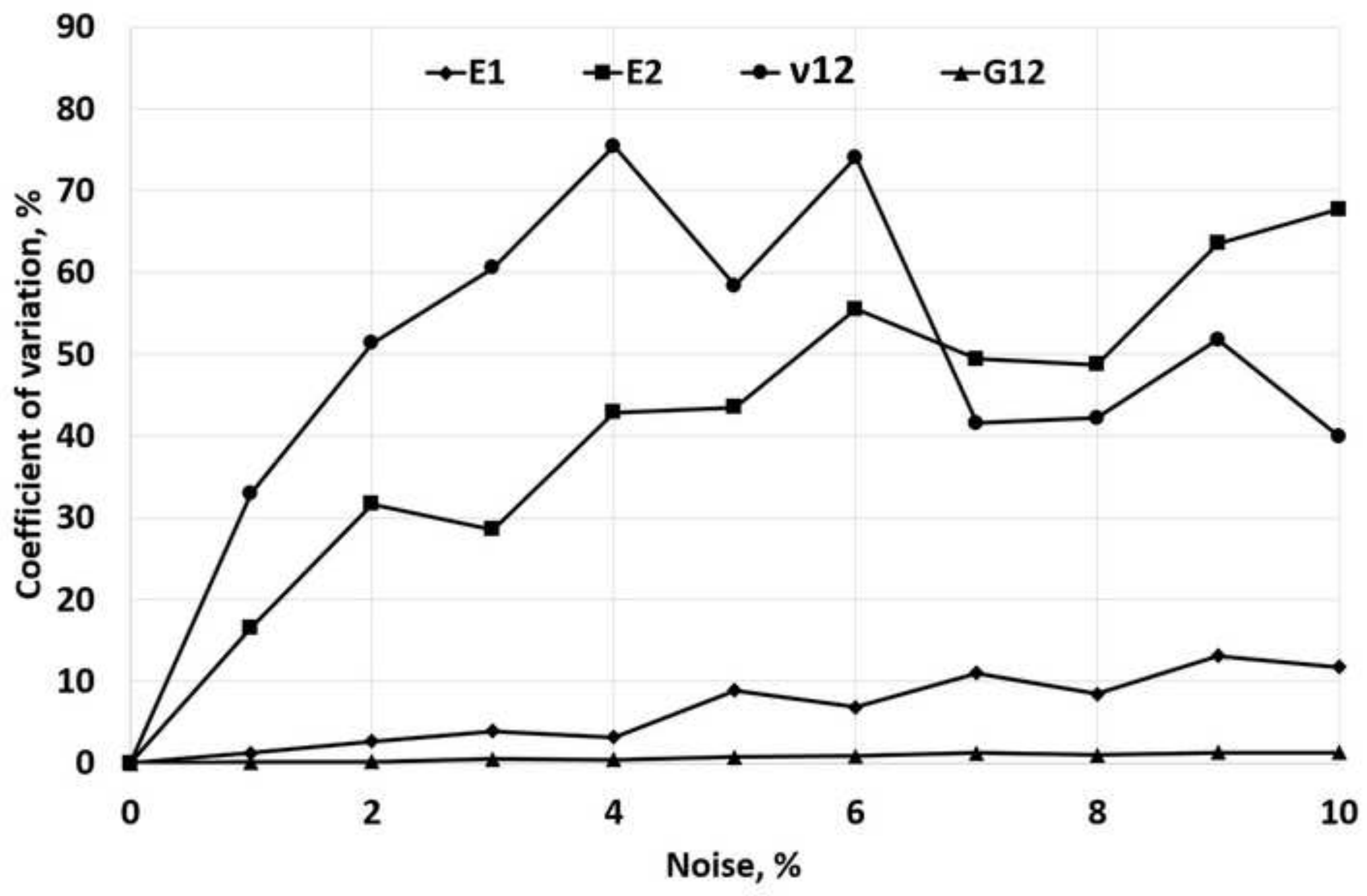




\section{Tables}

Table 1 Direct and inverse problems

\begin{tabular}{|c|c|c|c|}
\hline \multicolumn{2}{|c|}{ Direct problem } & \multicolumn{2}{c|}{ Inverse problem } \\
\hline Known & Unknown & Known & Unknown \\
\hline Geometry & $u$ & Geometry & \\
$\bar{u}$ on $S_{u}$ & $\sigma$ & $\bar{u}$ on $S_{u}$ & \\
$\bar{T}$ on $S_{T}$ & $\epsilon$ & $\tilde{u}$ on $\Gamma$ & \\
$C$ & & $\bar{T}$ on $S_{T}$ & \\
\hline
\end{tabular}

Table 2 Properties of Carbon Fiber Yarn, TAIRYFIL [20]

\begin{tabular}{|c|c|c|c|c|c|c|c|}
\hline Fiber Type & $\begin{array}{c}\text { Number of } \\
\text { Filaments }\end{array}$ & $\begin{array}{c}\text { Yield Tex } \\
(\mathrm{g} / 1000 \mathrm{~m})\end{array}$ & $\begin{array}{c}\text { Tensile } \\
\text { Strength } \\
(\mathrm{MPa})\end{array}$ & $\begin{array}{c}\text { Tensile } \\
\text { Modulus } \\
(\mathrm{GPa})\end{array}$ & $\begin{array}{c}\text { Elongation } \\
(\%)\end{array}$ & $\begin{array}{c}\text { Density } \\
\left(\mathrm{gm} / \mathrm{cm}^{3}\right)\end{array}$ & $\begin{array}{c}\text { Filament } \\
\text { Diameter } \\
(\mu \mathrm{m})\end{array}$ \\
\hline TC-36S & 12,000 & 800 & 4900 & 250 & 2.0 & 1.81 & 7 \\
\hline
\end{tabular}

Table 3 Properties of SWANCOR 2552 Epoxy resin [21]

\begin{tabular}{|c|c|c|c|c|c|c|c|}
\hline $\begin{array}{c}\text { Solid } \\
\text { Content } \\
(\%)\end{array}$ & $\begin{array}{c}\text { Density } \\
\left(\mathrm{gm} / \mathrm{cm}^{3}\right)\end{array}$ & $\begin{array}{c}\text { Viscosity } \\
(\mathrm{CPS})\end{array}$ & $\begin{array}{c}\text { Curing Time } \\
(\mathrm{min})\end{array}$ & $\begin{array}{c}\text { Tensile } \\
\text { Strength } \\
(\mathrm{MPa})\end{array}$ & $\begin{array}{c}\text { Tensile } \\
\text { Modulus } \\
(\mathrm{GPa})\end{array}$ & $\begin{array}{c}\text { Elongation } \\
(\%)\end{array}$ & $\begin{array}{c}\text { Density of } \\
\text { cured resin } \\
\left(\mathrm{gm} / \mathrm{cm}^{3}\right)\end{array}$ \\
\hline 65 & 1.02 & 200 & $\begin{array}{c}@ 120^{\circ} \mathrm{C}: 120 \\
@ 150^{\circ} \mathrm{C}: 60\end{array}$ & $70 \sim 90$ & $2.5^{\sim} 3.5$ & $3.5^{\sim} 4.5$ & $1.17 \sim 1.23$ \\
\hline
\end{tabular}

Table 4 Resin and fiber percentages in composite specimen

\begin{tabular}{|l|c|c|c|}
\hline & Resin & Fiber & Composite \\
\hline Density $\left(\mathrm{gm} / \mathrm{cm}^{3}\right)$ & 1.2 & 1.81 & 1.56 \\
\hline Weight fraction (\%) & 29 & 71 & - \\
\hline Volume fraction (\%) & 39 & 61 & - \\
\hline
\end{tabular}

Table 5 Elastic moduli of composite specimen from direct methods

\begin{tabular}{|l|c|c|c|c|}
\hline & $\mathrm{E}_{1}(\mathrm{GPa})$ & $\mathrm{E}_{2}(\mathrm{GPa})$ & $\mathrm{V}_{12}$ & $\mathrm{G}_{12}(\mathrm{GPa})$ \\
\hline Rule of mixtures & 151.2 & 7.4 & - & - \\
\hline Tensile tests & 145 & 8 & 0.31 & - \\
\hline Literature & - & - & - & 5 \\
\hline
\end{tabular}

only G12 is taken from literature [25] as this property was not determined from direct method

Table 6 Bounds on elastic constants for the optimization problem

\begin{tabular}{|l|c|c|c|c|}
\hline & $\mathrm{E}_{1}(\mathrm{GPa})$ & $\mathrm{E}_{2}(\mathrm{GPa})$ & $\mathrm{V}_{12}$ & $\mathrm{G}_{12}(\mathrm{GPa})$ \\
\hline Lower bound & 50 & 1 & 0.1 & 1 \\
\hline Upper bound & 350 & 15 & 0.5 & 15 \\
\hline
\end{tabular}


Table 7 Applied loads, initial guess in cost function minimization and estimated parameter values Case I

\begin{tabular}{|c|c|c|c|c|c|c|c|c|}
\hline Load (N) & \multicolumn{5}{|c|}{ Initial Guess } & \multicolumn{3}{c|}{ Estimated parameters } \\
\hline Initial load =5 & $\begin{array}{c}\mathrm{E}_{1} \\
(\mathrm{GPa})\end{array}$ & $\begin{array}{c}\mathrm{E}_{2} \\
(\mathrm{GPa})\end{array}$ & $\mathrm{V}_{12}$ & $\begin{array}{c}\mathrm{G}_{12} \\
(\mathrm{GPa})\end{array}$ & $\begin{array}{c}\mathrm{E}_{1} \\
(\mathrm{GPa})\end{array}$ & $\begin{array}{c}\mathrm{E}_{2} \\
(\mathrm{GPa})\end{array}$ & $\mathrm{V}_{12}$ & $\begin{array}{c}\mathrm{G}_{12} \\
(\mathrm{GPa})\end{array}$ \\
\hline 7 & 204.3 & 12.2 & 0.15 & 5.9 & 138.7 & 12.8 & 0.10 & 2.5 \\
\hline 9 & 69.1 & 5.7 & 0.32 & 8.0 & 165.4 & 12.9 & 0.19 & 1.0 \\
\hline 11 & 80.5 & 12.9 & 0.48 & 7.0 & 177.8 & 13.3 & 0.10 & 1.0 \\
\hline 13 & 77.5 & 7.2 & 0.46 & 8.0 & 137.9 & 13.0 & 0.10 & 1.0 \\
\hline 15 & 174.4 & 3.2 & 0.44 & 6.2 & 140.0 & 12.9 & 0.10 & 1.0 \\
\hline 17 & 193.6 & 10.5 & 0.26 & 2.9 & 162.7 & 11.6 & 0.25 & 1.0 \\
\hline 19 & 183.8 & 3.1 & 0.21 & 2.4 & 133.6 & 13.0 & 0.10 & 1.1 \\
\hline Target values & & & & & & & & \\
\hline & & & & & 145.0 & 8.0 & 0.31 & 5.0 \\
\hline Average & & & & & & & & \\
\hline Standard deviation & 58.5 & 4.1 & 0.12 & 2.4 & 17.3 & 0.5 & 0.06 & 0.6 \\
\hline & & & & & & & & \\
\hline Coefficient of variation (\%) & 36.8 & 50.5 & 40.2 & 49.8 & 11.5 & 4.2 & 45.8 & 44.7 \\
\hline Relative error (\%) & & & & & 4.0 & 59.8 & -56.7 & -75.3 \\
\hline
\end{tabular}


2017-03-09

\section{A projected finite element update method for inverse identification of material constitutive parameters in transversely isotropic laminates}

Siddiqui, Muhammad Zeeshan

Springer

Siddiqui, M., Khan, S.Z., Khan, M.A. et al. A projected finite element update method for inverse identification of material constitutive parameters in transversely isotropic laminates.

Experimental Mechanics, 2017, Vol 57, Iss 5, pp755-772

http://dx.doi.org/10.1007/s11340-017-0269-z

Downloaded from Cranfield Library Services E-Repository 